\title{
Research Article \\ Effects of Crack on Vibration Characteristics of Mistuned Rotated Blades
}

\author{
Hailong Xu, ${ }^{1}$ Zhongsheng Chen, ${ }^{1}$ Yongmin Yang, ${ }^{1}$ Limin Tao, ${ }^{1}$ and Xuefeng Chen ${ }^{2}$ \\ ${ }^{1}$ Science and Technology on Integrated Logistics Support Laboratory, National University of Defense Technology, Changsha, \\ Hunan 410073, China \\ ${ }^{2}$ State Key Laboratory for Manufacturing and Systems Engineering, Xian Jiaotong University, Xian, Shanxi 710049, China \\ Correspondence should be addressed to Zhongsheng Chen; chenzhongsheng@nudt.edu.cn
}

Received 19 October 2016; Accepted 16 January 2017; Published 15 February 2017

Academic Editor: Vadim V. Silberschmidt

Copyright (C) 2017 Hailong Xu et al. This is an open access article distributed under the Creative Commons Attribution License, which permits unrestricted use, distribution, and reproduction in any medium, provided the original work is properly cited.

Rotated blades are key mechanical components in turbine and high cycle fatigues often induce blade cracks. Meanwhile, mistuning is inevitable in rotated blades, which often makes it much difficult to detect cracks. In order to solve this problem, it is important and necessary to study effects of crack on vibration characteristics of mistuned rotated blades (MRBs). Firstly, a lumped-parameter model is established based on coupled multiple blades, where mistuned stiffness with normal distribution is introduced. Next, a breathing crack model is adopted and eigenvalue analysis is used in coupled lumped-parameter model. Then, numerical analysis is done and effects of depths and positions of a crack on natural frequency, vibration amplitude, and vibration localization parameters are studied. The results show that a crack causes natural frequency decease and vibration amplitude increase of cracked blade. Bifurcations will occur due to a breathing crack. Furthermore, based on natural frequencies and vibration amplitudes, variational factors are defined to detect a crack in MRBs, which are validated by numerical simulations. Thus, the proposed method provides theoretical guidance for crack detection in MRBs.

\section{Introduction}

Rotated blades are one of the main parts of turbine, which play important roles of energy conversion and often are called the heart of turbines. Low pressure turbine stages have long slender blades which undergo high bending deformation caused by natural frequencies in lower operating range. In order to limit vibratory deformations, these blades of low pressure stages are often stiffened with lacing wire connections. During working, rotated blades are often exposed to severe environments including high-speed rotating aerodynamic force, large centrifugal forces, and vibration transmission from other parts. In addition, some of them are affected by thermal stresses. Thus, rotated blades usually cause different kinds of faults under these extreme operation environments. In particular, blade cracks are one class of dangerous faults. If cracks cannot be detected as soon as possible, they can expand dramatically under the complex incentive conditions and thus lead to disastrous consequences [1-3]. Ideally, rotated blades generally are considered to be cyclic symmetry. In this case, a microcrack can cause vibration localization in tuned rotated blades $[4,5]$, so detection of microcracks became very easy in tuned rotated blades because they are sensitive to microcracks. However, manufacturing tolerances, using abrasion and material properties, can result in little differences between rotated blades. This phenomenon is usually called mistuning $[6,7]$. Vibration localization appears in MRBs and leads to high vibration stress concentrate in minority blades of MRBs, which can induce fatigue cracks in MRBs. When cracks appear in MRBs, they inevitably change mistuned characteristics and vibration characteristics of MRBs. So crack detection in MRBs is difficult to be reached, which is a necessary and important thing for operational safety of MRBs.

Up to now, many methods have been studied to detect and identify cracks in rotated blades. Vibration monitoring is a common and effective method for damage identification which requires us to fully understand the effects of cracks on vibration characteristics of rotated blades. So vibration characteristics of rotated blades and effects of crack on 
vibration characteristics of rotated blades are hot research issues. Many scholars have done lots of researches. Kim and Stubbs [8] presented a practical methodology to nondestructively localize cracks and estimate the sizes of the cracks in beam-type structures using changes in frequencies. Piovan and Sampaio [9] used continuous parameters model to analyze rotated beams with functionally graded properties and quantificationally studied vibration characteristics of the blade based on centrifugal force. The common characteristic of the above researches is that an isolated blade model is constructed and analyzed. So the model is simple and can get vibration characteristics of a rotated blade quickly. But rotated blades are interconnected with each other by lacing wire, and vibration characteristics of MRBs are different from a single inevitable blade. So it is inappropriate to use isolated blade model to analyze vibration characteristics of MRBs.

Some scholars further propel related researches. They used coupled multiple-blades dynamic model to analyze vibration characteristics of rotated blades. Prohl [10] was the first to analyze blade group dynamics using lumpedparameter modeling of blades with lacing wire attached to the blade tip. Here, lacing wires were considered as spring elements and forced vibration was computed using Holzer Method. This analytical method computes intricately for blades with varying cross section and twist along the length. Finite element model is well suited for such complex problems and it was used by Bajaj [11] to determine the natural frequencies of packeted blade in coupled bendingtorsion modes. Lim et al. [12] have carried out an extensive modal analysis based on energy integral for the multipacket blade system considering disk flexibility, angular speed, and shroud flexibility. The above researched works in this segment are done based on ideal blades. In other words, blades have been considered to be identical, both geometrically and structurally. Such ideal type of blades is called tuned blades. The tuned condition preserves symmetry in the mathematical model. However, in practice, the cyclic symmetry of blades is inevitably destroyed by manufacturing tolerance, using abrasion and properties of materials. So the analytical results have a little difference compared with actual rotated blades.

Next, some related researches considered mistuning of blades and used mistuned model to study vibration behaviors of rotated blades. Chandrashaker and Adhikari [13] proposed the Modal Assurance Criterion Localization Factor which may help designers to easily obtain crucial information, such as the degree of localization for all the vibration modes in the system. Wang et al. [14] analyzed resonance characteristics of blisk under random mistuning. And localization of low order bending mode was most greatly impacted by random mistuning. Hai et al. [15] studied the effects of different mistuning factor on the natural frequency and vibration characteristics of blisk. It was found that quality and stiffness of blades were more sensitive to mistuning than damping of blades. It is mature that rotated blades are analyzed based on mistuning. In the operation of MRBs, vibration induces high cycle fatigue and then leads to cracks. It is a difficult and important problem of cracks affecting vibration characteristics of MRBs and related researches are studied rarely. But some scholars study effects of crack on vibration

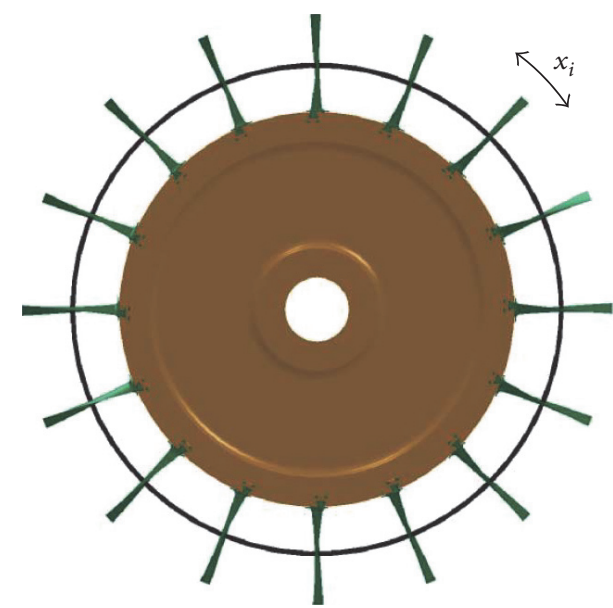

FIgURE 1: The 3D model of MRBs.

characteristics of tuned rotated blades. Huang $[4,5]$ studied the effect of a number of blades and distribution of cracks on vibration localization in a cracked pretwisted blade system. Hou [16] presented a study of the mechanisms of crackinginduced mistuning in bladed disks. An analytical model was formulated and then analyzed to understand the relationship between the blade dynamic response and the crack length.

In the aforementioned researches, vibration characteristics of MRBs and effects of crack on tuned rotated blades are studied by some scholars. However, practical experience shows that mistuning is inherent feature of rotated blades and crack can change vibration characteristics of MRBs by change mistuning of MRBs. To the best of our knowledge, rare works have been reported in this area. In this paper, coupled multiple-blades model is built based on inherent mistuning in rotated blades and then used to analyze the effects of crack on vibration characteristics of MRBs. Moreover, analytical results are used to study crack detection in MRBs. The left contents are organized as follows: In Section 2, a simplified model is formulated and then analyzed to understand vibration characteristics of MRBs with a breathing crack. In addition, a local parameter and a variational factor are introduced to quantificationally describe vibration characteristics of MRBs. In Section 3, modal characteristics of MRBs are analyzed by numerical analysis. Next, the effects of depths and positions of a crack on natural frequency, vibration amplitude, and localization parameters of vibration response (LPVRs) of MRBs are studied. Based on natural frequencies and vibration amplitudes, variational factors are used to detect a crack in MRBs. Then, nonlinear behaviors of LPVRs are explained by bifurcation diagram of vibration displacement. Finally, conclusions are marked in Section 4.

\section{Modeling and Analysis of MRBs with a Breathing Crack}

The 3D model of MRBs is shown in Figure 1. Rotated blades are fixed on central disk, which are long slender blades and undergo high bending deformation caused by natural frequencies in lower operating range. These blades 


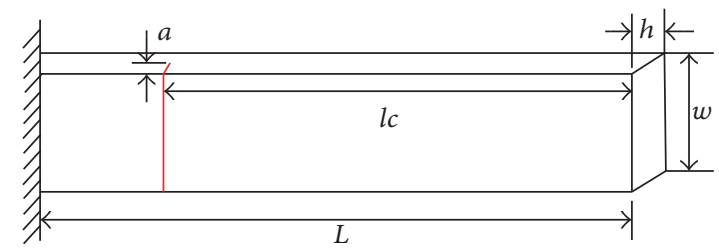

FIgURE 2: Schematic diagram of the equivalent cantilever beam with a crack.

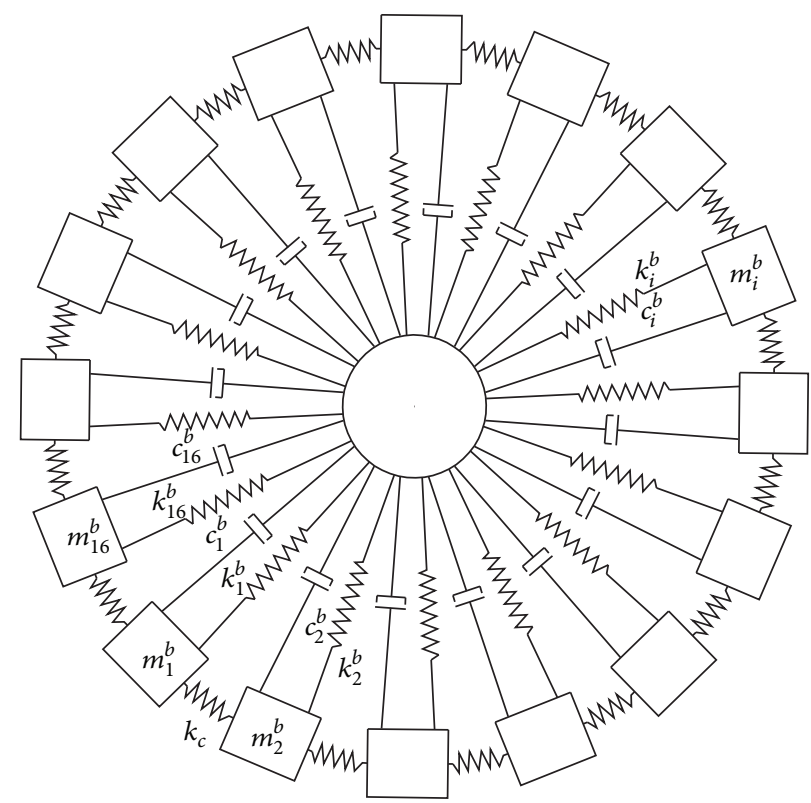

FIGURE 3: An equivalent lumped-parameter model of coupled MRBs.

go through transient resonant condition at nozzle passing frequency [17]. In order to limit vibratory deformations, these blades are often stiffened with lacing wire connections. In addition, micromistuning occurs between each blade due to manufacturing tolerances, using abrasion and material properties. Mistuning can be changed as fatigue cracks appeared in MRBs during operational life. These can change vibration characteristics of MRBs. Therefore, crack detection in MRBs can be realized probably through changes of vibration characteristics. So model of MRBs is built first and then is used to study the effects of crack on vibration characteristics of MRBs. For the sake of simplicity, the disk is assumed to be rigid and a blade in Figure 1 is simplified to cantilever beam in Figure 2, which is conducive to establish and solve followup model. Length, width, and height of the blade in Figure 2 are denoted as $L, w$, and $h$, respectively. When a crack appears in a blade, the distance between the crack and the blade tip is denoted as $l c$, and the depth of the crack is denoted as $a$. Then, we make an investigation into modal characteristics of tuned blades and MRBs using a lumped-parameter model approach similar to [16]. The simplified lumped-parameter model is shown in Figure 3. In this model, every blade is represented by a single-degree-of-freedom (SDOF) lumped-parameter model with the equivalent stiffness $k_{i}^{b}$ and the equivalent damping $c_{i}^{b}$. The equivalent mass $m_{i}^{b}$ is concentrated at the blade tip. Lacing wire between two adjacent blades adds stiffness to blades; hence, it is modeled as a massless spring and stiffness is $k_{c} . i$ is the serial number of a blade and the total number of blades is $n$, so $i=1,2, \ldots, n$. Lumped-parameter model is adopted here mainly to facilitate parametric study, particularly investigating the effect of lessened stiffness in a blade due to a crack. The objective is primarily to study changes of vibration characteristics in MRBs for different possibilities of cracks, and then expect to instruct crack detection in MRBs.

Vibration equations of coupled MRBs based on airflow excitation in Figure 3 can be written as follows:

$$
\begin{aligned}
& m_{1}^{b} \ddot{x}_{1}+c_{1}^{b} \dot{x}_{1}+k_{1}^{b} x_{1}+k_{c}\left(x_{1}-x_{16}\right) \\
& +k_{c}\left(x_{1}-x_{2}\right)=F_{1} \\
& \vdots \\
& m_{i}^{b} \ddot{x}_{i}+c_{i}^{b} \dot{x}_{i}+k_{i}^{b} x_{i}+2 k_{c} x_{i}-k_{c} x_{i-1}-k_{c} x_{i+1}=F_{i} \\
& \vdots \\
& m_{16}^{b} \ddot{x}_{16}+c_{16}^{b} \dot{x}_{16}+k_{16}^{b} x_{16}+k_{c}\left(x_{16}-x_{15}\right) \\
& +k_{c}\left(x_{16}-x_{1}\right)=F_{16} .
\end{aligned}
$$


2.1. Modal Analysis of MRBs with a Breathing Crack. Based on simplified model of MRBs, (1) can be written as follows:

$$
\mathbf{M X}+\mathbf{C} \dot{\mathbf{X}}+\mathbf{K X}=\mathbf{F},
$$

where

$$
\begin{aligned}
& \mathbf{M}=\left[\begin{array}{ccccc}
m_{1}^{b} & 0 & \cdots & 0 & 0 \\
0 & m_{2}^{b} & \cdots & 0 & 0 \\
\cdots & \cdots & \cdots & \cdots & \cdots \\
0 & 0 & \cdots & m_{15}^{b} & 0 \\
0 & 0 & \cdots & 0 & m_{16}^{b}
\end{array}\right], \\
& \mathbf{C}=\left[\begin{array}{ccccc}
c_{1}^{b} & 0 & \cdots & 0 & 0 \\
0 & c_{2}^{b} & \cdots & 0 & 0 \\
\cdots & \cdots & \cdots & \cdots & \cdots \\
0 & 0 & \cdots & c_{15}^{b} & 0 \\
0 & 0 & \cdots & 0 & c_{16}^{b}
\end{array}\right] \\
& \mathbf{K}=\left[\begin{array}{ccccc}
k_{1}^{b}+2 k_{c} & -k_{c} & \cdots & 0 & -k_{c} \\
-k_{c} & k_{2}^{b}+2 k_{c} & -k_{c} & 0 & 0 \\
\cdots & \ldots & \cdots & \ldots & \ldots \\
0 & 0 & -k_{c} & k_{15}^{b}+2 k_{c} & -k_{c} \\
-k_{c} & 0 & \cdots & -k_{c} & k_{16}^{b}+2 k_{c}
\end{array}\right] \text {, } \\
& \mathbf{X}=\left[\begin{array}{c}
x_{1} \\
x_{2} \\
\cdots \\
x_{15} \\
x_{16}
\end{array}\right],
\end{aligned}
$$$$
\mathbf{F}=\left[\begin{array}{c}
F_{1} \\
F_{2} \\
\cdots \\
F_{15} \\
F_{16}
\end{array}\right] .
$$

$\mathbf{M}, \mathbf{C}$ and $\mathbf{K}$ are equivalent mass matrix, equivalent external damping matrix, and equivalent stiffness matrix, which are symmetric matrixes. $\mathbf{X}$ and $\mathbf{F}$ are vibration displacements of all blades and external airflow forces. When $\mathbf{F}$ act on MRBs, the governing equations of motion are coupled ordinary differential equations of second order. Firstly, in natural modal analysis of MRBs, damping matrix $\mathbf{C}$ is not considered and the external forces $\mathbf{F}=\mathbf{0}$ [18]. So (2) is converted into

$$
\mathbf{M X}+\mathbf{K X}=\mathbf{0} .
$$

We set solution of (4) as $\mathbf{X}(t)=\boldsymbol{\varphi} \sin (\omega t+\theta)$, where $\omega$ and $\theta$ are scalars and $\boldsymbol{\varphi}$ is vector. By substituting $\mathbf{X}(t)$ into (4), one can get the following formula:

$$
\left(\mathbf{K}-\omega^{2} \mathbf{M}\right) \boldsymbol{\varphi}=\mathbf{0} .
$$

In mathematical problem, (5) is a generalized eigenvalue problem of matrixes $\mathbf{M}$ and $\mathbf{K}$. Here, $\omega$ and $\boldsymbol{\varphi}$ are eigenvalues and eigenvectors, respectively. In mechanical system, $\omega$ and $\varphi$ are natural frequencies and natural shapes in simplified model of rotated blades. If $\varphi$ have nonzero solutions, necessary and sufficient conditions must be satisfied as in the following equation:

$$
\operatorname{det}\left(\mathbf{K}-\omega^{2} \mathbf{M}\right)=0
$$

which can be written in a normalized form as

$$
\left|\begin{array}{ccccc}
k_{1}^{b}+2 k_{c}-\omega^{2} m_{1}^{b} & -k_{c} & \ldots & 0 & -k_{c} \\
-k_{c} & k_{2}^{b}+2 k_{c}-\omega^{2} m_{2}^{b} & -k_{c} & 0 & 0 \\
\ldots & \ldots & \ldots & \ldots & \ldots \\
0 & 0 & -k_{c} & k_{15}^{b}+2 k_{c}-\omega^{2} m_{15}^{b} & -k_{c} \\
-k_{c} & 0 & \ldots & -k_{c} & k_{16}^{b}+2 k_{c}-\omega^{2} m_{16}^{b}
\end{array}\right|=0 .
$$

In tuned condition, equivalent stiffness of each blade is defined as $k_{i}^{b}=k_{b}$ and equivalent mass $m_{i}^{b}=m_{b}$. According to [19], equivalent stiffness and equivalent mass of a static cantilever beam in lumped-parameter model are calculated as $k_{\mathrm{eq}}^{\text {static }}=E I \pi^{4} / 32 L^{3}$ and $m_{b}=0.228 m^{\prime} L$, where $E$ is Young's modulus of blades, $I$ is rotary inertia of a blade, which can be calculated as $I=w h^{3} / 12, m^{\prime}$ is the unit length mass of a blade, which can be calculated as $m^{\prime}=\rho w h ; \rho$ is density of blades.
Centrifugal rigidity will appear in rotated blades due to centrifugal force. When lumped-parameter model is modeled and analyzed, equivalent stiffness of rotated blades is considered at different rotated speed. According to [20], equivalent stiffness of a rotated blade at different speed can be calculated as follows:

$$
k_{b}=k_{\mathrm{eq}}^{\text {static }}+4 \pi^{2} m_{b} B D^{2}
$$


where $D$ is rotated speed of blades. $B$ is the calibration factor, which can be calculated as follows:

$$
B=\left(\frac{\pi}{30}\right)^{2} \frac{\int_{0}^{L}(H+y)\left[\int_{0}^{y}(d z / d y)^{2} d y\right] d y}{\int_{0}^{L} z^{2} d y},
$$

where $H$ is the radius of the disc. $z=\left(\sin \lambda_{r} y-\operatorname{sh} \lambda_{r} y\right)+$ $\zeta_{r}\left(\cos \lambda_{r} y-\operatorname{ch} \lambda_{r} y\right)$ with $\zeta_{r}=\left(\cos \beta_{r}+\operatorname{ch} \beta_{r}\right) /\left(\sin \beta_{r}-\operatorname{sh} \beta_{r}\right)$ and $\lambda_{r}=\beta_{r} / L . r$ is the order of mode. $z$ depends on vibration mode of the blade. The first-order mode is considered in SDOF lumped-parameter model. So we will have $\beta_{r}=\beta_{1}=$ 1.875 .

Coupled stiffness between two adjacent blades is calculated as follows:

$$
k_{c}=R k_{b}
$$

where $R$ is coupled factor. Using $k_{i}^{b}=k_{b}, m_{i}^{b}=m_{b}$, and (10), (7) can be simplified as in the following form:

$$
\begin{aligned}
& \left|\begin{array}{ccccc}
1+2 R-\frac{\omega^{2}}{\omega_{b}^{2}} & -R & \ldots & 0 & -R \\
-R & 1+2 R-\frac{\omega^{2}}{\omega_{b}^{2}} & -R & 0 & 0 \\
\ldots & \ldots & \ldots & \ldots & \ldots \\
0 & 0 & -R & 1+2 R-\frac{\omega^{2}}{\omega_{b}^{2}} & -R \\
-R & 0 & \ldots & -k_{c} & 1+2 R-\frac{\omega^{2}}{\omega_{b}^{2}}
\end{array}\right| \\
& =0,
\end{aligned}
$$

where $\omega_{b}=\sqrt{k_{b} / m_{b}}$. Equation (11) is expanded and solved to obtain natural frequencies of tuned blades. Solutions are shown as follows:

$$
\begin{aligned}
\omega_{i \text { tuning }}^{2}=\omega_{b}^{2} & {\left[1+2 R\left(1-\cos \frac{2 \pi(k-1)}{n}\right)\right], } \\
& \quad k=1,2,2, \ldots, 8,8,9 ; i=1,2, \ldots, 15,16 .
\end{aligned}
$$

Evidently, except for $k=1$ or $k=9$, double eigenvalues occur. By substituting (12) into (5), this equation is solved and solutions of $\varphi_{i}^{\text {tuning }}$ exist in the following form:

$$
\begin{aligned}
& \boldsymbol{\varphi}_{i}^{k}=\left[1, \cos \alpha_{k}, \ldots, \cos (n-1) \alpha_{k}\right] \\
& \boldsymbol{\varphi}_{i}^{k}=\left[0, \sin \alpha_{k}, \ldots, \sin (n-1) \alpha_{k}\right], \\
& \quad k=1,2,2,3,3, \ldots, 8,8,9 ; n=16,
\end{aligned}
$$

where $\alpha_{k}=2 \pi(k-1) / n$. Based on (12) and (13), we can get natural frequencies and natural shapes of tuned rotated blades.

As mistuning is inevitable in rotated blades, mistuned stiffness is used to represent micromistuning of MRBs [21]. We define the mistuned factor of each blade as $\delta_{i}$, so the equivalent stiffness of $i$ th blade is shown as follows:

$$
k_{i}^{b}=\left(1+\delta_{i}\right) k_{b} .
$$

On the other hand, equivalent mass does not have mistuning and $m_{i}^{b}=m_{b}$. By substituting (14) and $m_{i}^{b}=m_{b}$ into (7), one can get the following formula:

$$
\left|\begin{array}{ccccc}
\left(1+\delta_{1}\right) k_{b}+2 k_{c}-\omega^{2} m_{b} & -k_{c} & \cdots & 0 & -k_{c} \\
-k_{c} & \left(1+\delta_{2}\right) k_{b}+2 k_{c}-\omega^{2} m_{b} & -k_{c} & 0 & 0 \\
\ldots & \ldots & \cdots & \ldots & \cdots \\
0 & 0 & -k_{c} & \left(1+\delta_{15}\right) k_{b}+2 k_{c}-\omega^{2} m_{b} & -k_{c} \\
-k_{c} & 0 & \cdots & -k_{c} & \left(1+\delta_{16}\right) k_{b}+2 k_{c}-\omega^{2} m_{b}
\end{array}\right|=0
$$

Next, (15) can be simplified as follows:

$$
\left|\begin{array}{ccccc}
\left(1+\delta_{1}\right)+2 R-\frac{\omega^{2}}{\omega_{b}^{2}} & -R & \cdots & 0 & -R \\
-R & \left(1+\delta_{2}\right)+2 R-\frac{\omega^{2}}{\omega_{b}^{2}} & -R & 0 & \ldots \\
\ldots & \cdots & \ldots & \cdots & \cdots \\
\ldots & 0 & -R & \left(1+\delta_{15}\right)+2 R-\frac{\omega^{2}}{\omega_{b}^{2}} & -R \\
-R & 0 & \cdots & -R & \left(1+\delta_{16}\right)+2 R-\frac{\omega^{2}}{\omega_{b}^{2}}
\end{array}\right|=0 .
$$


Then, (16) can be expanded and solved to obtain natural frequencies. Solutions are shown as follows:

$$
\begin{array}{r}
\omega_{\text {imistuning }}^{2}=\omega_{b}^{2}\left[1+\delta_{i}+2 R\left(1-\cos \frac{2 \pi(k-1)}{n}\right)\right], \\
\quad k=1,2,2, \ldots, 8,8,9 ; i=1,2,3, \ldots, 16,
\end{array}
$$

when $\omega_{i \text { mistuning }}^{2}$ is chosen of different value, substituting it into (5). The equation is solved and $\varphi_{i}^{\text {mistuning }}$ is got. So we can get natural frequencies and natural shapes of MRBs.

When a crack appears in MRBs, contact area of the crack periodically opened and closed due to vibration of the cracked blade, which is called a breathing crack. Consequently, equivalent stiffness of the cracked blade is timevarying and changed period is related to airflow excitation $F_{i}$. Next, the key problem is how to obtain equivalent timevarying stiffness of a blade with a breathing crack. According to [19], a cosine function is adopted to represent time-varying stiffness of the blade with a breathing crack; that is,

$$
k_{i}^{\mathrm{eq}}(t)=k_{\text {iopen }}^{b}+\frac{1}{2}\left(k_{i \text { close }}^{b}-k_{i \text { open }}^{b}\right)(1+\cos (\omega t)),
$$

where $k_{i \text { close }}^{b}$ and $k_{i \text { open }}^{b}$ are equivalent stiffness of the blade with a crack fully open and close state, respectively.

When a crack keeps fully close state in a blade, equivalent stiffness of the blade $k_{i \text { close }}^{b}=k_{i}^{b}$; if the crack keeps fully open state in the blade, equivalent stiffness of the cracked blade can be obtained as follows [19]:

$$
k_{\text {iopen }}^{b}=\frac{1}{\left(1 / k_{i}^{b}+\left(72 l c^{2} \pi\left(1-v^{2}\right) / E w h^{4}\right) g(\alpha)\right)},
$$

where $g(\alpha)=a^{2}\left(19.60 \alpha^{8}-40.69 \alpha^{7}+47.04 \alpha^{6}-32.99 \alpha^{5}+\right.$ $\left.20.30 \alpha^{4}-9.98 \alpha^{3}+4.60 \alpha^{2}-1.05 \alpha+0.63\right), \alpha=a / h$, and $v$ is Poisson's ratio of MRBs. Obviously, $k_{i o p e n}^{b}$ is closely related to depth and position of a crack. By substituting (14) and (18) into (7), one can get the following formula:

$$
\begin{array}{ccccc}
\left(1+\delta_{1}\right) k_{b}+2 k_{c}-\omega^{2} m_{b} & -k_{c} & \ldots & 0 & -k_{c} \\
-k_{c} & \left(1+\delta_{2}\right) k_{b}+2 k_{c}-\omega^{2} m_{b} & -k_{c} & 0 & 0 \\
\vdots & \vdots & \vdots & \vdots & \vdots \\
0 & -k_{c} & k_{i}^{\mathrm{eq}}(t)+2 k_{c}-\omega^{2} m_{b} & -k_{c} & \cdots \\
\vdots & \vdots & \vdots & \vdots & \vdots \\
-k_{c} & 0 & -k_{c} & \left(1+\delta_{15}\right) k_{b}+2 k_{c}-\omega^{2} m_{b} & -k_{c} \\
- & 0 & \cdots & -k_{c} & \left(1+\delta_{16}\right) k_{b}+2 k_{c}-\omega^{2} m_{b}
\end{array}
$$

$=0$.

Due to equivalent time-varying stiffness of $i$ th blade with a breathing crack, $k_{i}^{\mathrm{eq}}(t)+2 k_{c}-\omega^{2} m_{b}$ appears in (20). So we cannot solve (20) and get natural frequencies of MRBs with a breathing crack. Under this case, according to [22], natural frequency of a blade with a breathing crack can be calculated as follows:

$$
\omega_{i}^{b}=\frac{2 \omega_{i \text { close }}^{b} \omega_{i \text { open }}^{b}}{\omega_{i \text { close }}^{b}+\omega_{i \text { open }}^{b}}
$$

where $\omega_{i c l o s e}^{b}, \omega_{i \text { open }}^{b}$ are natural angular frequency of the blade with a crack fully open and close state, respectively. Based on (7), we can obtain eigenvalue equation of MRBs with a fully open crack as follows:

$$
\begin{array}{|ccccc}
\left(1+\delta_{1}\right) k_{b}+2 k_{c}-\omega^{2} m_{b} & -k_{c} & \cdots & 0 & -k_{c} \\
-k_{c} & \left(1+\delta_{2}\right) k_{b}+2 k_{c}-\omega^{2} m_{b} & -k_{c} & 0 & 0 \\
\vdots & \vdots & \vdots & \vdots & \vdots \\
0 & -k_{c} & k_{i \mathrm{open}}^{b}+2 k_{c}-\omega^{2} m_{b} & -k_{c} & \cdots \\
\vdots & \vdots & \vdots & \vdots & \vdots \\
-k_{c} & 0 & -k_{c} & \left(1+\delta_{15}\right) k_{b}+2 k_{c}-\omega^{2} m_{b} & -k_{c} \\
& 0 & \cdots & -k_{c} & \left(1+\delta_{16}\right) k_{b}+2 k_{c}-\omega^{2} m_{b}
\end{array} \mid
$$

$=0$. 
Using the above equation, $\omega_{i \text { open }}^{b}$ can be calculated. Combining with $\omega_{\text {iclose }}^{b}$ from (17), we can get natural frequencies of MRBs with a breathing crack by (21). Then, substituting it into (5), the equation is solved and gets natural shapes $\varphi_{i}^{\text {crack }}$ under different natural frequencies.

2.2. Forced Vibration Modeling and Analysis of MRBs with a Breathing Crack. In practice, vibrations of MRBs are mainly caused by airflow excitation. Simultaneously, system damping dissipates vibration energy of MRBs. So, in the next analysis, damping effect is considered in forced vibration by airflow excitation. In the above modal analysis, mistuned stiffness is used to represent micromistuning of MRBs and $m_{i}^{b}=m_{b}$. In addition, equivalent damping of MRBs does not cause mistuning and $c_{i}^{b}=c_{b}$. It can be calculated as $c_{b}=2 \xi m_{b} \omega_{b}$, where $\xi$ is damping ratio. Forced vibration equations of MRBs by airflow excitation $\mathbf{F}$ are shown as (1). In regard to airflow excitation of rotated blades, many researchers agree that airflow force under steady flow has cyclicity in rotated blades. So harmonic function can be used to represent equivalent model of airflow force [23, 24]. Airflow excitation of each blade can be simplified and airflow force of $i$ th blade is shown as follows:

$$
F_{i}=F_{0} \cos \left(\omega_{\text {excite }} t+\frac{2 \pi E_{0}(i-1)}{n}\right)
$$

where $F_{0}$ is the equivalent amplitude of airflow force. $\omega_{\text {excite }}$ is the angular frequency of airflow force, which can be calculated as $\omega_{\text {excite }}=2 \pi D / 60 . E_{0}$ is the order of airflow force.

As to $m_{i}^{b}=m_{b}$ and $c_{i}^{b}=c_{b}$, we can obtain

$$
\mathbf{C}=\alpha \mathbf{M}
$$

where $\alpha$ is constant. According to (24), which is known as proportional damping, by substituting (24) into (2), we obtain

$$
\mathbf{M} \ddot{\mathbf{X}}+\alpha \mathbf{M} \dot{\mathbf{X}}+\mathbf{K X}=\mathbf{F} .
$$

By expressing the solution vector $\mathbf{X}$ as a linear combination of the natural shapes of the undamped system, we obtain the following equation:

$$
\mathbf{X}=\left[\boldsymbol{\varphi}_{i}^{\text {mistuning }}\right] \mathbf{q},
$$

where $\mathbf{q}$ is modal coordinate. Next, by substituting (26) into (25), (25) can be rewritten as follows:

$$
\begin{aligned}
\mathbf{M} & {\left[\boldsymbol{\varphi}_{i}^{\text {mistuning }}\right] \ddot{\mathbf{q}}+\alpha \mathbf{M}\left[\boldsymbol{\varphi}_{i}^{\text {mistuning }}\right] \dot{\mathbf{q}}+\mathbf{K}\left[\boldsymbol{\varphi}_{i}^{\text {mistuning }}\right] \mathbf{q} } \\
& =\mathbf{F} .
\end{aligned}
$$

Premultiplication of (27) by $\left[\boldsymbol{\varphi}_{i}^{\text {mistuning }}\right]^{T}$ leads to

$$
\begin{aligned}
& {\left[\boldsymbol{\varphi}_{i}^{\text {mistuning }}\right]^{T} \mathbf{M}\left[\boldsymbol{\varphi}_{i}^{\text {mistuning }}\right] \ddot{\mathbf{q}}} \\
& \quad+\alpha\left[\boldsymbol{\varphi}_{i}^{\text {mistuning }}\right]^{T} \mathbf{M}\left[\boldsymbol{\varphi}_{i}^{\text {mistuning }}\right] \dot{\mathbf{q}} \\
& \quad+\left[\boldsymbol{\varphi}_{i}^{\text {mistuning }}\right]^{T} \mathbf{K}\left[\boldsymbol{\varphi}_{i}^{\text {mistuning }}\right] \mathbf{q}=\left[\boldsymbol{\varphi}_{i}^{\text {mistuning }}\right]^{T} \mathbf{F} .
\end{aligned}
$$

Based on modal analysis results of MRBs, (28) can be reduced to

$$
\begin{aligned}
I \ddot{\mathbf{q}}+ & \alpha I \dot{\mathbf{q}} \\
& +\operatorname{diag}\left[\omega_{1 \text { mistuning }}^{2}, \omega_{2 \text { mistuning }}^{2}, \ldots, \omega_{16 \text { mistuning }}^{2}\right] \mathbf{q} \\
= & {\left[\boldsymbol{\varphi}_{i}^{\text {mistuning }}\right]^{T} \mathbf{F} . }
\end{aligned}
$$

According to (29), we can obtain that

$$
\begin{aligned}
\ddot{q}_{i}(t)+\alpha \dot{q}_{i}(t)+\omega_{\text {imistuning }}^{2} q_{i}(t)=Q_{i}( & \\
& \\
& i=1,2, \ldots, 16,
\end{aligned}
$$

where $\omega_{\text {imistuning }}$ is natural frequency of $i$ th blade under undamped system and $\mathbf{Q}(t)=\left[\boldsymbol{\varphi}_{i}^{\text {mistuning }}\right]^{T} \mathbf{F}$. We solve the above decoupled second-order linear differential equations (30). Then, $\mathbf{X}$ is obtained by substituting $\left[q_{i}(t)\right]$ into (26). In other words, forced vibration response of every blade by airflow excitation has been solved.

When a breathing crack appears in MRBs,

$$
\mathbf{K}=\left[\begin{array}{ccccc}
\left(1+\delta_{1}\right) k_{b}+2 k_{c} & -k_{c} & \cdots & 0 & -k_{c} \\
-k_{c} & \left(1+\delta_{1}\right) k_{b}+2 k_{c} & -k_{c} & 0 & 0 \\
\vdots & \vdots & \vdots & \vdots & \vdots \\
\cdots & -k_{c} & k_{i}^{\mathrm{eq}}(t) & -k_{c} & \cdots \\
\vdots & \vdots & \vdots & \vdots & \vdots \\
0 & 0 & -k_{c} & \left(1+\delta_{1}\right) k_{b}+2 k_{c} & -k_{c} \\
-k_{c} & 0 & \cdots & -k_{c} & \left(1+\delta_{1}\right) k_{b}+2 k_{c}
\end{array}\right]
$$


So (29) is converted into the following formula:

$$
\begin{aligned}
I \ddot{\mathbf{q}} & +\alpha I \dot{\mathbf{q}}+\operatorname{diag}\left[\omega_{1}^{2}, \omega_{2}^{2}, \ldots, \mu \frac{k_{i}^{\mathrm{eq}}(t)}{m_{b}}, \ldots, \omega_{16}^{2}\right] \mathbf{q} \\
& =\left[\boldsymbol{\varphi}_{i}\right]^{T} \mathbf{F},
\end{aligned}
$$

where $\mu$ come from computational process. We solve the above decoupled second-order nonlinear differential equations (32). Then, combining with (26), we can get vibration response of MRBs with a breathing crack.

The equivalent time-varying stiffness of cracked blade changes compared with other rotated blades due to a crack. Then, the crack can change original mistuning of MRBs. No matter what original micromistuning or mistuning change due to a crack, these can change vibration characteristics of MRBs. So a quantitative parameter is needed to represent vibration characteristics of MRBs, which is used to analyze effects of crack on vibration characteristics of MRBs. From [25], a local parameter is introduced to achieve this goal. In simplified lumped-parameter model, vibration energy of a blade is proportional to square of amplitude. Then, we can use Euclidean norm to define the local parameter as follows:

$$
L=\sqrt{\frac{X_{\max }^{2}-(1 /(n-1)) \sum_{i=1, i \neq j}^{n} X_{i}^{2}}{(1 /(n-1)) \sum_{i=1, i \neq j}^{n} X_{i}^{2}}},
$$

where $X_{i}$ is the amplitude of $i$ th blade. $j$ is the serial number of maximum amplitude among rotated blades and the value is $X_{\max }$. Equation (33) represents difference between the maximum vibration energy of a blade and the average vibration energy of other blades. So the local parameter describes vibration characteristics of all blades. In order to describe vibration characteristics of every blade, which are used for crack detection in MRBs, a variational factor is defined and shown as follows:

$$
\delta \theta=\frac{\left|\theta_{2}-\theta_{1}\right|}{\theta_{1}},
$$

where $\theta_{1}$ is a vibration parameter of a blade under reference condition. $\theta_{2}$ is a vibration parameter of the blade by a crack changing. We can use the variational factor to study vibration parameter change of every blade by a crack.

Next, we will use these models and parameters to analyze vibration characteristics of MRBs and the corresponding effects of crack. In the meantime, crack detection in MRBs is studied based on analytical results.

\section{Numerical Analysis of Crack on Vibration Characteristics of MRBs}

Numerical analyses are done in this section based on previous foundations. Materials and geometric properties of rotated blades in numerical analysis are listed in Table 1. In Section 1, manufacturing tolerances, using abrasion and material properties, are mainly reasons of mistuning in rotated blades. Characteristics of these factors are random in
TABLE 1: Materials and geometric properties of rotated blades.

\begin{tabular}{lcc}
\hline Symbols & Properties & Value \\
\hline$L$ & Length of the blade $(\mathrm{mm})$ & 95 \\
$h$ & Thickness of the blade $(\mathrm{mm})$ & 2.5 \\
$w$ & Width of the blade $(\mathrm{mm})$ & 45 \\
$H$ & Radius of the disc $(\mathrm{mm})$ & 200 \\
$E$ & Young's modulus $(\mathrm{Pa})$ & $0.689 \times 10^{11}$ \\
$\nu$ & Poisson's ratio & 0.33 \\
$\rho$ & Density of the blade $\left(\mathrm{kg} / \mathrm{m}^{3}\right)$ & 2800 \\
$\xi$ & Damping ratio & 0.01 \\
$n$ & Number of blades & 16 \\
$D$ & Rotated speed of MRBs $(\mathrm{RPM})$ & 5000 \\
$E_{0}$ & Order of airflow force & 1 \\
\hline
\end{tabular}

TABLE 2: Values of mistuned factors.

\begin{tabular}{lccc}
\hline Mistuned factors & Mistuning1 & Mistuning2 & Mistuning3 \\
\hline$\delta_{1}$ & -0.017632 & -0.030061 & -0.110847 \\
$\delta_{2}$ & -0.003227 & 0.002358 & 0.033806 \\
$\delta_{3}$ & -0.008396 & 0.017452 & -0.011001 \\
$\delta_{4}$ & -0.016606 & 0.018437 & 0.036723 \\
$\delta_{5}$ & 0.030125 & 0.003265 & -0.020770 \\
$\delta_{6}$ & 0.015009 & -0.016293 & -0.051720 \\
$\delta_{7}$ & -0.003899 & 0.008074 & -0.000349 \\
$\delta_{8}$ & 0.001149 & 0.015123 & 0.009334 \\
$\delta_{9}$ & 0.003589 & 0.043689 & -0.016968 \\
$\delta_{10}$ & -0.000121 & -0.001876 & -0.058778 \\
$\delta_{11}$ & -0.005704 & -0.005884 & 0.048257 \\
$\delta_{12}$ & 0.009340 & -0.010096 & 0.007893 \\
$\delta_{13}$ & -0.011383 & -0.038540 & -0.028275 \\
$\delta_{14}$ & 0.019572 & 0.010428 & 0.048655 \\
$\delta_{15}$ & -0.001027 & -0.041088 & 0.014687 \\
$\delta_{16}$ & -0.003233 & -0.005398 & -0.050701 \\
Average value & 0 & 0 & 0 \\
Standard deviation & $1 \%$ & $2 \%$ & $4 \%$ \\
\hline
\end{tabular}

practice. According to [25], mistuned factors $\delta_{i}$ comply with normal distribution. In this paper, the mistuned factors $\delta_{i}$ under different mistuned levels are listed in Table 2.

3.1. Effects of Mistuning and Coupling on Modal Characteristics of MRBs. In order to testify vibrational differences of tuned rotated blades and MRBs, firstly, numerical analyses are done here based on (13) and $\varphi_{i}^{\text {mistuning }}$. Materials and geometric properties of blades in simulations are listed in Table 1. Coupled factor $R$ is chosen as 0.01 . Natural shapes of tuned blades (mistuned factors $\delta_{i}=0$ ) under different orders are shown as Figure 4. First-order natural shapes are equivalent and other orders' natural shapes change harmoniously in Figure 4. In MRBs, mistuned factors $\delta_{i}$ are chosen as mistuning1 in Table 2. Other parameters remain constant. Natural shapes of MRBs are shown in Figure 5. From the figure, 

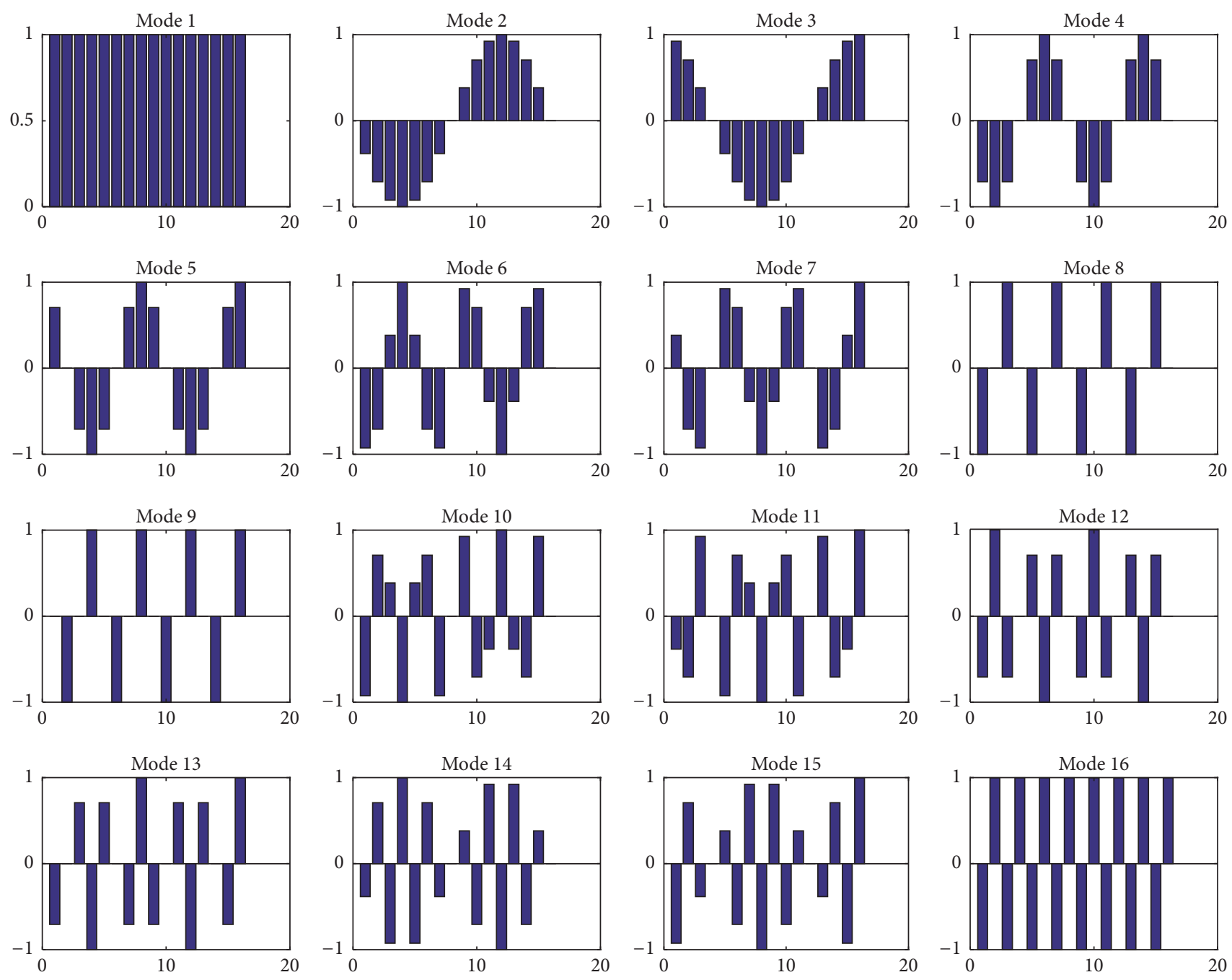

FIGURE 4: Natural shapes of tuned rotated blades under different orders.

modal amplitude mainly appears on a few blades for every order. This phenomenon is called mode localization and it is caused by mistuning $[4,5,16,26,27]$. In order to show mode localization of MRBs quantificationally, (33) is used in the next analysis. Then, we will study effects of mistuning and coupling on modal characteristics of MRBs further.

Based on Figure 5, mode localization parameters (MLPs) can be got combined with $\varphi_{i}^{\text {mistuning }}$ and (33). In addition, natural frequencies of MRBs are from (17). Firstly, effects of mistuning on mode localization of MRBs are studied. Materials and geometric properties of blades remain constant and $R=0.01$. Natural frequencies and MLPs change with orders under different mistuned levels are calculated and shown in Figures 6 and 7. According to (12), tuned blades have superposition of natural frequency except for those of first order and sixteenth order (e.g., natural frequency of second order is equal to that of third order and all of these are $289.8 \mathrm{~Hz}$ ) in Figure 6. Natural frequencies of all orders distribute in small range $(289.6 \mathrm{~Hz}-295.3 \mathrm{~Hz})$ of tuned rotated blades. But when mistuning is brought in rotated blades, superposition of natural frequencies is split. The range of natural frequencies increases with mistuning strengthening. In addition, natural frequency of first order decreases significantly with mistuning strengthening. In Figure 7, MLPs of first order and sixteenth order are 0 and MLPs of other orders are 1. This phenomenon is consistent with the results of Figure 4 . But with mistuning of MRBs strengthening (standard deviation of $\delta_{i}$ increasing), MLPs increase accordingly and MLP of first order increases obviously. The greater the dispersion of natural frequencies is, the less the occurrence of resonance appears in MRBs. So mistuned level of MRBs should be greater. On the other hand, biggish mistuning can cause MLPs of MRBs increasing in Figure 7, which induce fatigue cracks in MRBs easily. So mistuning of MRBs should be kept in appropriate range based on the above factors $[25,28]$.

Next, effects of coupling between MRBs $(R)$ on mode localization are studied further. Keep above parameters constant. Natural frequencies and MLPs change with orders under different coupled factors are calculated and shown in 

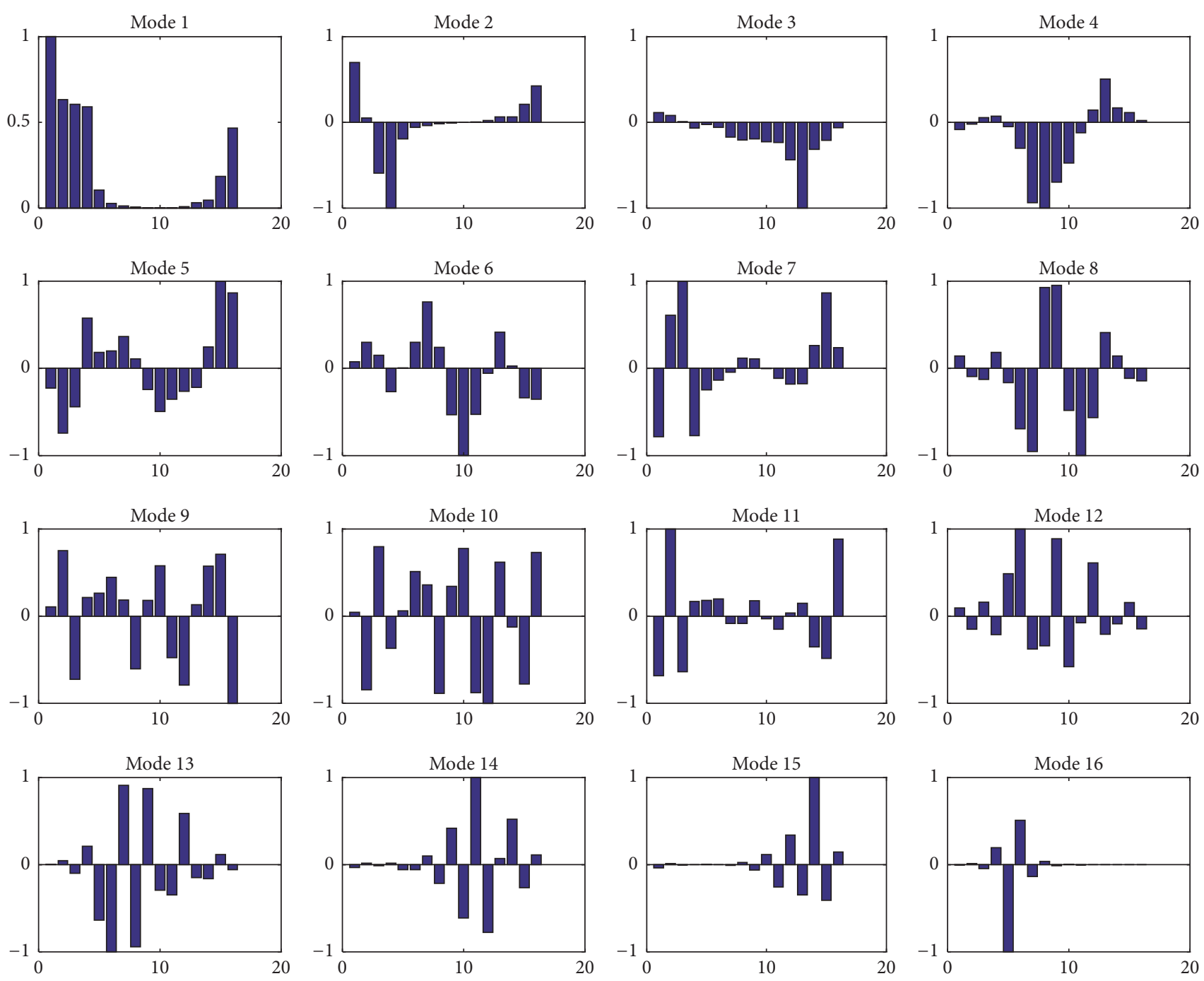

FIGURE 5: Natural shapes of MRBs under different orders.

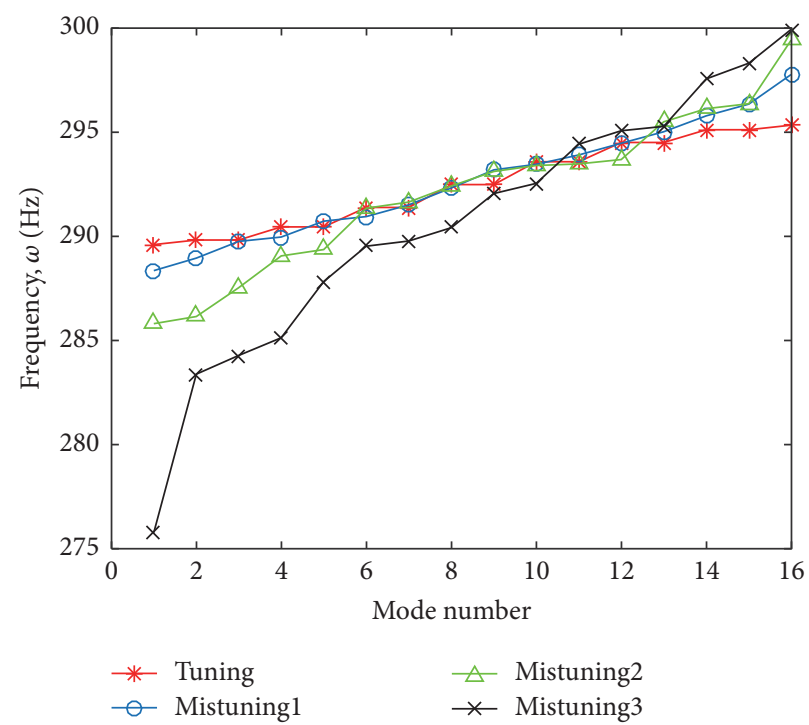

FIgURE 6: Natural frequencies of MRBs under different mistuned levels. 


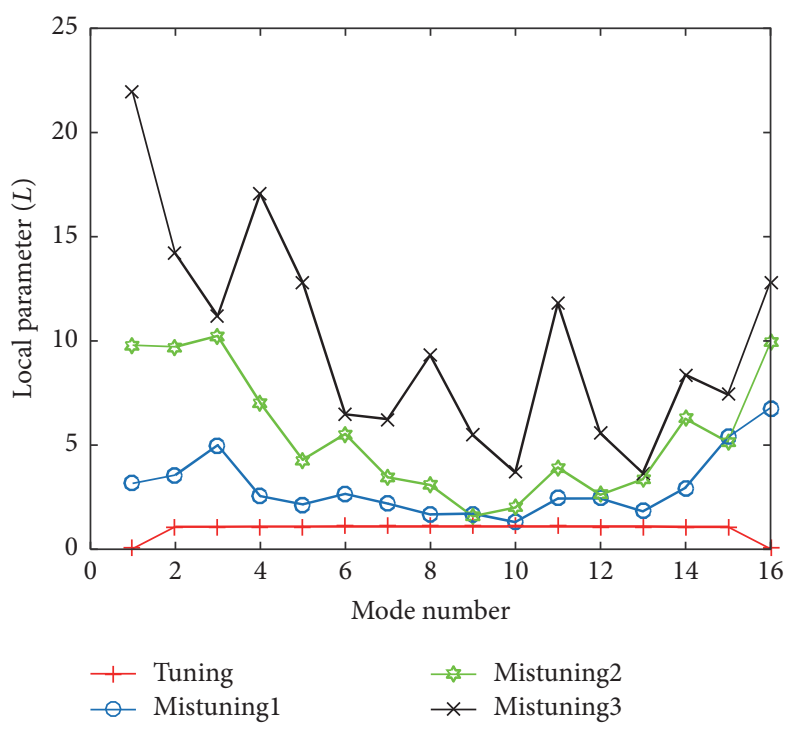

FIGURE 7: MLPs of MRBs under different mistuned levels.

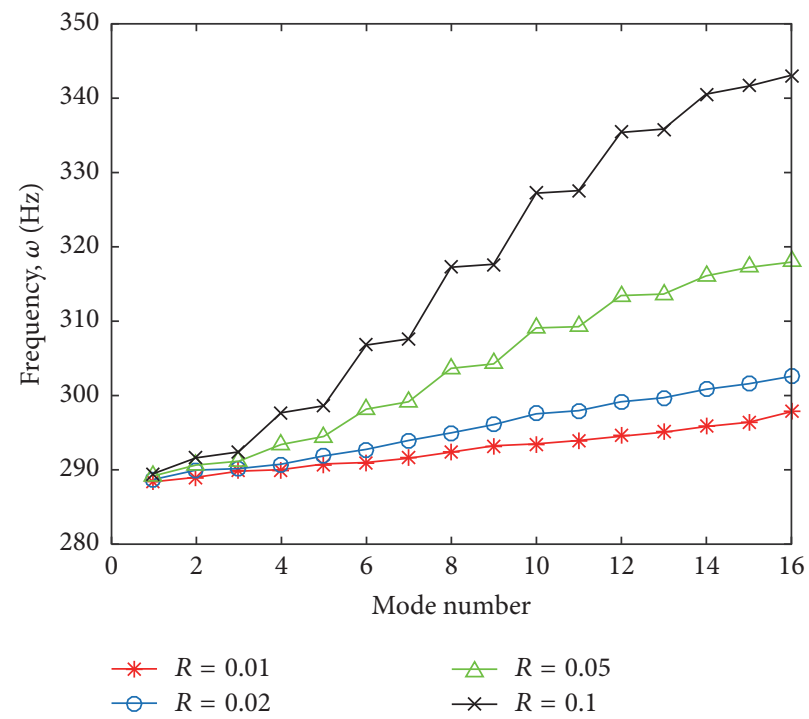

FIGURE 8: Natural frequencies of MRBs under different $R$.

Figures 8 and 9. In Figure 8, the range of natural frequencies increases with $R$ enhancing. When $R$ increases to 0.1 , superposition of natural frequencies reappears. In addition, natural frequency of last order increases markedly with coupled factor enhancing. In Figure 9, MLPs decrease contrary to $R$ enhancing and MLP of last order decreases obviously. When $R$ is greater than or equal to 0.05 , MLPs of all orders are almost equal to 1 . So mode localization of MRBs mainly appears in weak coupling pattern. The greater the dispersion of natural frequencies to avoid resonance in MRBs is, the smaller the MLPs are required. So it is particularly important to increase coupled level of MRBs. But, in practice, too large coupled level can potentially lead to resonance of blades and disk. So $R$ also has an upper limit $[25,28]$. In subsequent analysis of this paper, $R$ is no more than 0.1 .
3.2. Effects of a Crack on Modal Characteristics of MRBs. Based on the above analysis, we know modal characteristics of MRBs and get effects of mistuning and coupling on modal characteristics of MRBs. But when a crack appears in MRBs, mistuning of MRBs will be changed by the crack expanding. This can change vibration characteristics of MRBs. Next, we study quantificationally that depths and positions of a crack change natural frequencies, vibration amplitudes, and localization parameters of vibration response (LPVRs) of MRBs. These have important guidance for cracks detection in MRBs.

Firstly, we study effects of a crack on natural frequency of each blade in MRBs. In next analysis, natural frequencies of MRBs with a breathing crack are computed based on (21). In numerical analysis, the crack is assumed to locate at the 


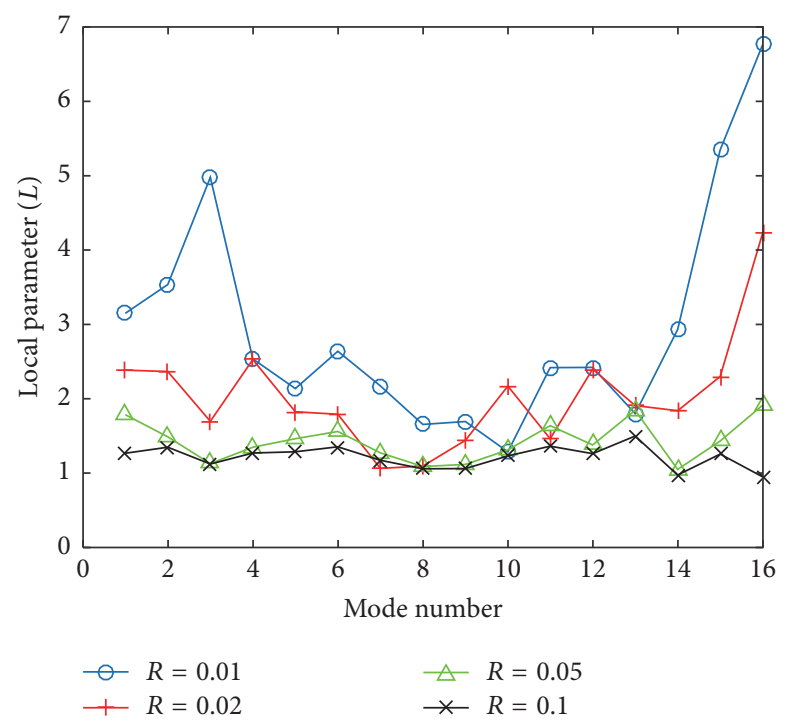

Figure 9: MLPs of MRBs under different $R$.

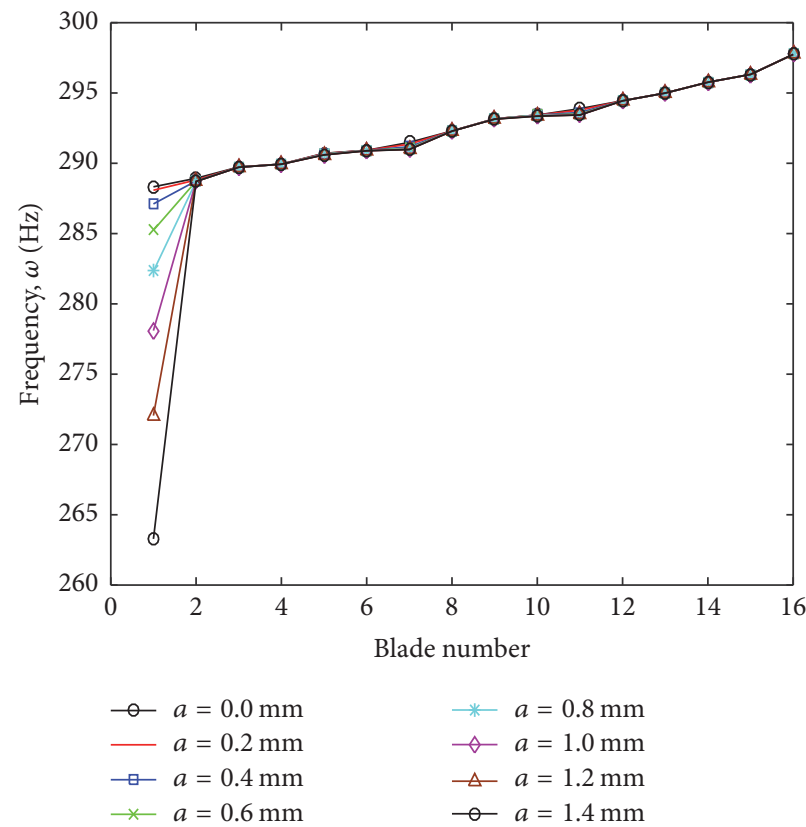

FIGURE 10: Natural frequencies of MRBs under different $a$.

1st blade. Materials and geometric properties of blades are listed in Table 1. Mistuned factors $\delta_{i}$ are chosen as mistuning1 in Table 2. Here, $R$ is chosen as 0.1 . Distance between the crack and 1st blade tip $l c$ is set as $85 \mathrm{~mm}$. Natural frequency of each blade under different depths of the crack $a$ is shown in Figure 10. From the figure, natural frequency of 1st blade decreases with $a$ increasing. Moreover, natural frequencies of other blades change because blades are interconnected with each other by lacing wire. In practice, we cannot determine which blade manifests a crack by natural frequency changes of MRBs. But we use $\delta \omega=\left|\omega_{2}-\omega_{1}\right| / \omega_{1}$ to analyze frequency change of each blade under different $a$, where $\omega_{1}, \omega_{2}$ are natural frequencies of a blade with different $a$, respectively. The analytical results are shown in Figure 11. In the figure, $L_{1}, L_{2}, L_{3}, L_{4}$ are frequency changes of all blades with $a=$ $0 \mathrm{~mm}$ and $a=0.1 \mathrm{~mm}, a=0.2 \mathrm{~mm}$ and $a=0.25 \mathrm{~mm}$, $a=0.9 \mathrm{~mm}$ and $a=0.91 \mathrm{~mm}$, and $a=1.5 \mathrm{~mm}$ and $a=$ $1.51 \mathrm{~mm}$, respectively. We conclude that frequency change of the cracked blade is obviously greater than other blades. Comparing with $L_{3}$ and $L_{4}$, when $a$ increases, frequency change increases as identical depth change of the crack. In practice, when a crack appears in MRBs, $a$ increases with MRBs continuous working. We can choose natural frequency of each blade at different period to analyze variational factor. 


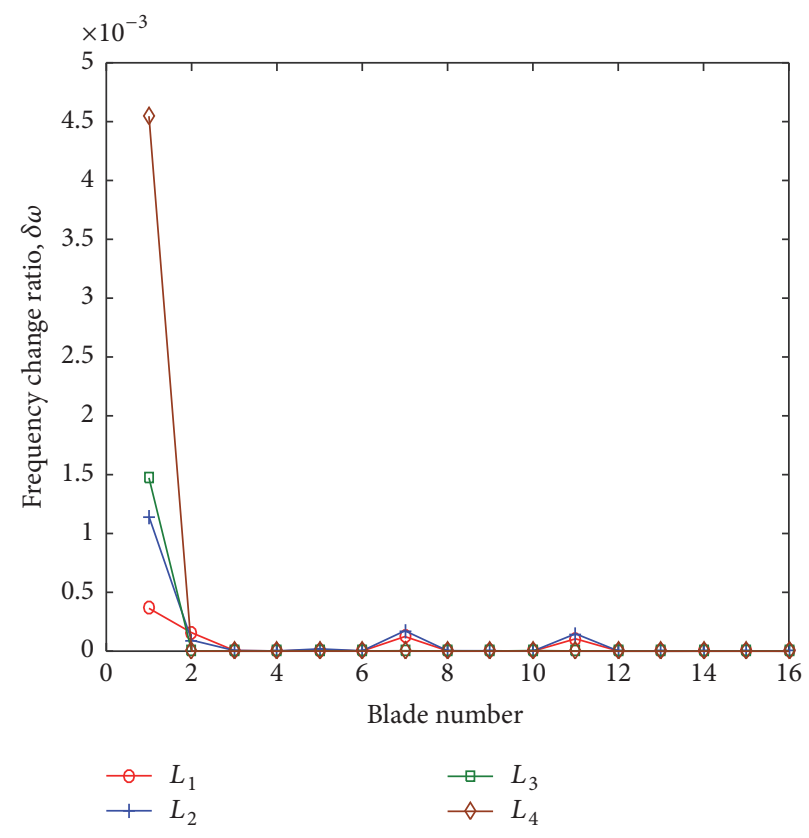

FIgURE 11: Frequencies change of MRBs under different $a$.

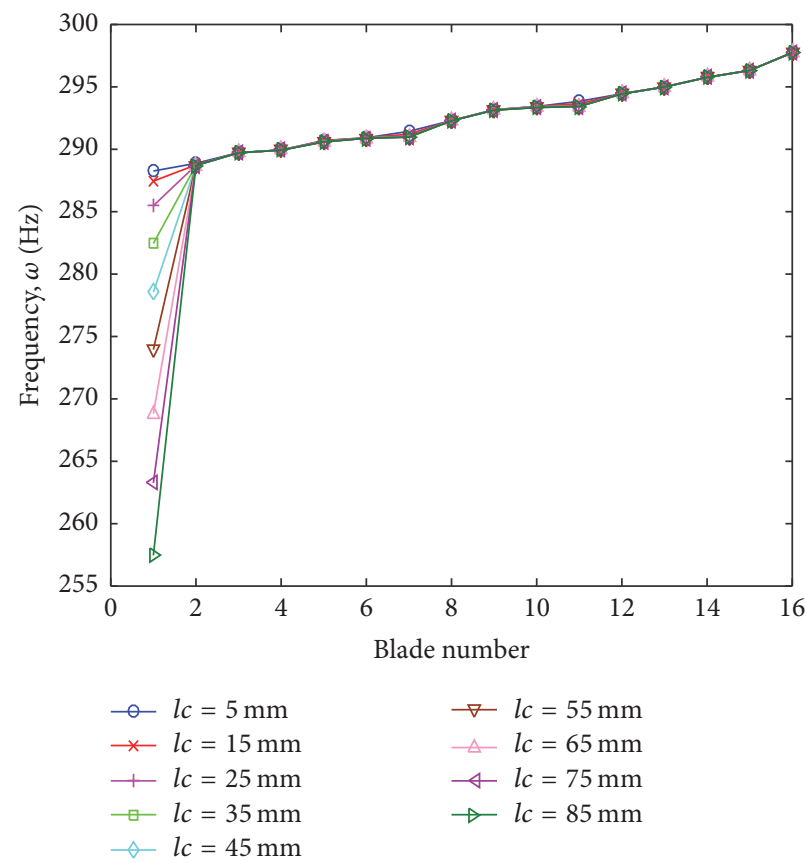

FIGURE 12: Natural frequencies of MRBs under different $l c$.

If natural frequencies of blades change, and variational factor of a blade is always larger than other blades obviously, the blade of MRBs can be initially determined as a cracked blade.

Next, we further study the effects of positions of a crack on natural frequencies of MRBs. Here, $a=1.5 \mathrm{~mm}$ and other parameters are fixed as in the above. The relations between positions of a crack and natural frequency of each blade are shown in Figure 12. When the crack is close to root of 1st blade, natural frequencies of 1st blade and other blades decrease. The $\delta \omega=\left|\omega_{2}-\omega_{1}\right| / \omega_{1}$ is used to analyze effects of the crack at different positions on frequency change of each blade, where $\omega_{1}, \omega_{2}$ are natural frequencies of a blade with different positions of a crack, respectively. The analytical results are shown in Figure 13. $L_{5}, L_{6}, L_{7}, L_{8}$ are frequency changes of all blades with $l c=5 \mathrm{~mm}$ and $l c=15 \mathrm{~mm}$, $l c=5 \mathrm{~mm}$ and $l c=40 \mathrm{~mm}, l c=5 \mathrm{~mm}$ and $l c=65 \mathrm{~mm}$, 


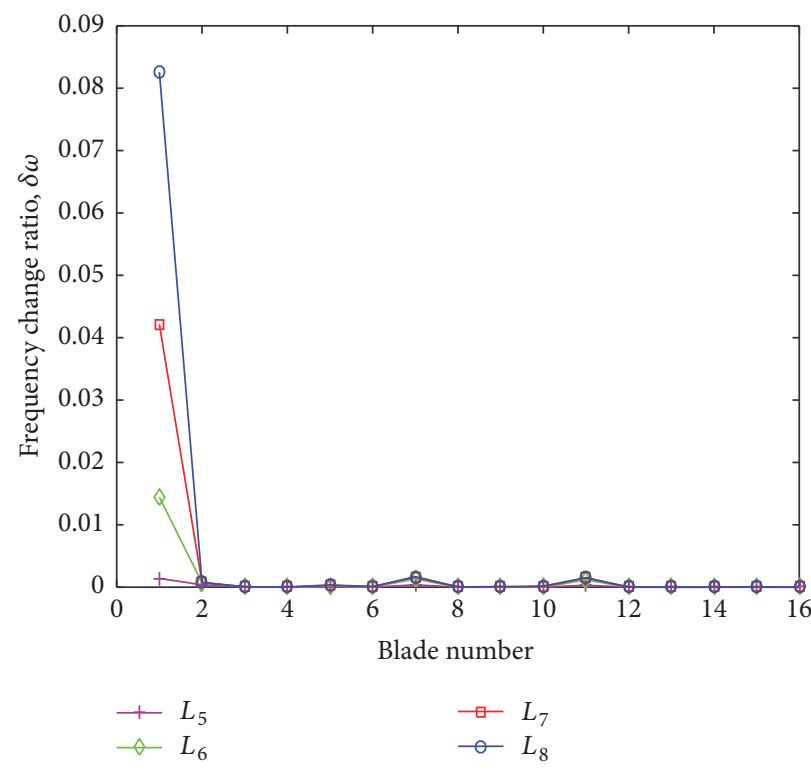

FIGURE 13: Frequencies change of MRBs under different $l c$.

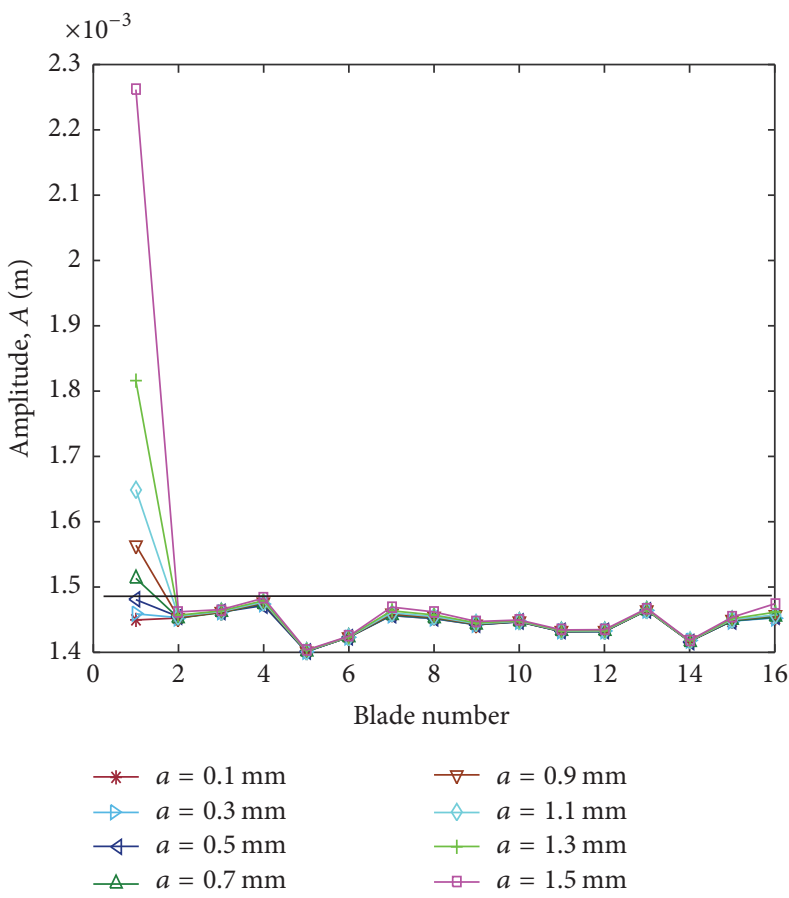

Figure 14: Vibration amplitudes of MRBs under different $a$.

and $l c=5 \mathrm{~mm}$ and $l c=90 \mathrm{~mm}$, respectively. We analyze frequency change of each blade between different positions of the crack and $l c=5 \mathrm{~mm}$. These can be seen that (i) when the crack is close to root of the cracked blade, frequency change of the cracked blade is obviously larger than other blades. (ii) Natural frequency of a blade changes by a crack in root, which is far greater than the crack in tip of the blade. In other words, when a crack appears in root of a blade, it is easy to be detected.
3.3. Effects of a Crack on Forced Vibration Response of $M R B s$. Based on the above analysis of a crack on modal characteristics of MRBs, the crack also changes vibration response of MRBs. Materials and geometric properties of blades are listed in Table 1. Mistuned factors $\delta_{i}$ are chosen as mistuning1 in Table 2 . Here, $F_{0}=30 \mathrm{~N}, R=0.01$, and $l c=$ $85 \mathrm{~mm}$. A crack is located at 1st blade; vibration amplitudes of MRBs under different $a$ are shown in Figure 14. From the figure, vibration amplitude of 1st blade increases along 


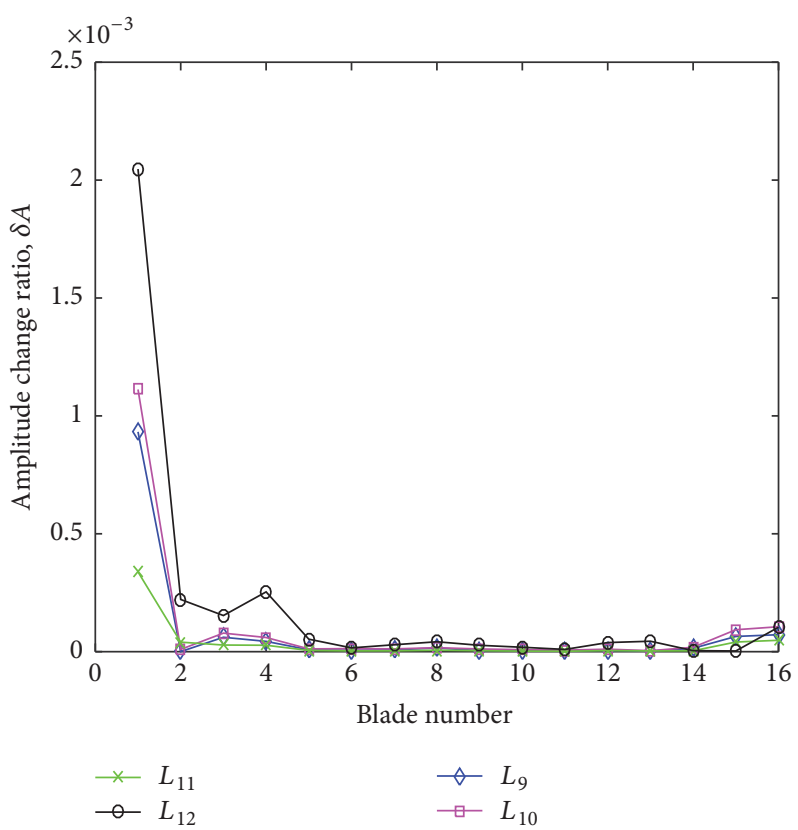

FIGURE 15: Vibration amplitude changes of MRBs under different $a$.

with $a$ increasing. But when $a \leq 0.5 \mathrm{~mm}$, the incremental amplitude is very small and vibration amplitude of the cracked blade is equal to maximum vibration amplitude. Furthermore, vibration amplitudes of other blades change with $a$ increasing. So we cannot determine which blade manifests a crack by vibration amplitude changes of MRBs in operation. Then, based on the above analytical results, we use $\delta A=\left|A_{2}-A_{1}\right| / A_{1}$ to analyze vibration amplitude change of each blade under different $a$, where $A_{1}, A_{2}$ are vibration amplitudes of a blade with different $a$, respectively. When $a \leq 0.5 \mathrm{~mm}$, the analytical results are shown in Figure 15. In the figure, $L_{9}, L_{10}, L_{11}, L_{12}$ are vibration amplitude changes of all blades with $a=0 \mathrm{~mm}$ and $a=0.1 \mathrm{~mm}, a=0.1 \mathrm{~mm}$ and $a=0.15 \mathrm{~mm}, a=0.2 \mathrm{~mm}$ and $a=0.21 \mathrm{~mm}$, and $a=0.25 \mathrm{~mm}$ and $a=0.3 \mathrm{~mm}$, respectively. Vibration amplitude change of the cracked blade is obviously greater than other blades. Comparing with $L_{10}$ and $L_{12}$, when $a$ increases, vibration amplitude change increases as identical depth change of the crack. In practice, when a crack appears in MRBs, $a$ expands with MRBs continuous working. We choose vibration amplitudes of rotated blades at different period to analyze variational factors. If vibration amplitudes of blades change and variational factor of a blade is always obviously larger than other blades, the blade of MRBs can be initially determined as a cracked blade. Moreover, when vibration amplitude of a blade is obviously larger than other blades, we also determine a cracked blade in MRBs.

Next, effects of positions of a crack on vibration amplitudes of MRBs are studied. Here, $a=1.5 \mathrm{~mm}$ and other parameters are fixed as in the above. Relations between vibration amplitudes and positions of the crack are shown in Figure 16. From the figure, vibration amplitude of 1st blade increases along with position of the crack close to root of 1st blade. But when $l c \leq 20 \mathrm{~mm}$, incremental amplitude of the cracked blade is very small and vibration amplitude of the cracked blade is equal to maximum vibration amplitude. In addition, vibration amplitudes of other blades change with $l c$ increasing. So cracks are detected hardly by vibration amplitude changes of MRBs. Then, vibration amplitude changes of all blades under different positions of a crack are shown in Figure 17. From the figure, $L_{13}, L_{14}, L_{15}, L_{16}$ are vibration amplitude changes of MRBs with $l c=1 \mathrm{~mm}$ and $l c=2 \mathrm{~mm}$, $l c=10 \mathrm{~mm}$ and $l c=10.5 \mathrm{~mm}, l c=15 \mathrm{~mm}$ and $l c=15.2 \mathrm{~mm}$, and $l c=19 \mathrm{~mm}$ and $l c=19.5 \mathrm{~mm}$, respectively. These can be seen that (i) when a crack appears in MRBs, vibration amplitude change of the cracked blade is larger than other blades. (ii) When a crack is close to root of the cracked blade, vibration amplitude change under identical position change of the crack is obviously larger than the crack in tip of the blade. In other words, when a crack appears in root of a blade, it is easily to be detected.

Based on the above analyses between parameters of the crack and vibration amplitudes of MRBs, we study effects of a crack to LPVRs further. Keep the above parameters constant, combining with (33) and vibration amplitudes of MRBs. The relations between LPVRs and parameter changes of a crack are shown in Figure 18. From the figure, we can summarize some conclusions as follows: (i) LPVRs almost do not change when depth of the crack is small and the crack appears in blade tip within a small range. (ii) LPVR increases obviously when the crack makes the vibration amplitude of the cracked blade greater than other blades. In addition, peak value appears in increased process. The main reason is the crack which is external crack and modeled as a breathing crack. Vibration amplitude of the cracked blade shows nonlinear phenomenon with parameters of the crack 


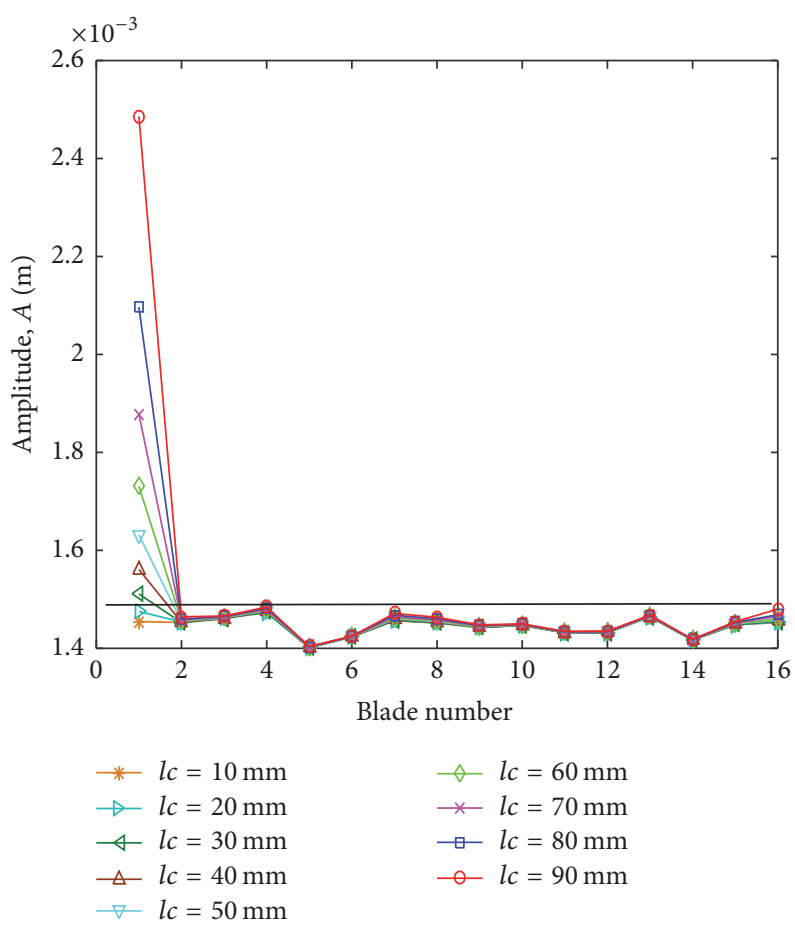

FIgURE 16: Vibration amplitudes of MRBs under different $l c$.

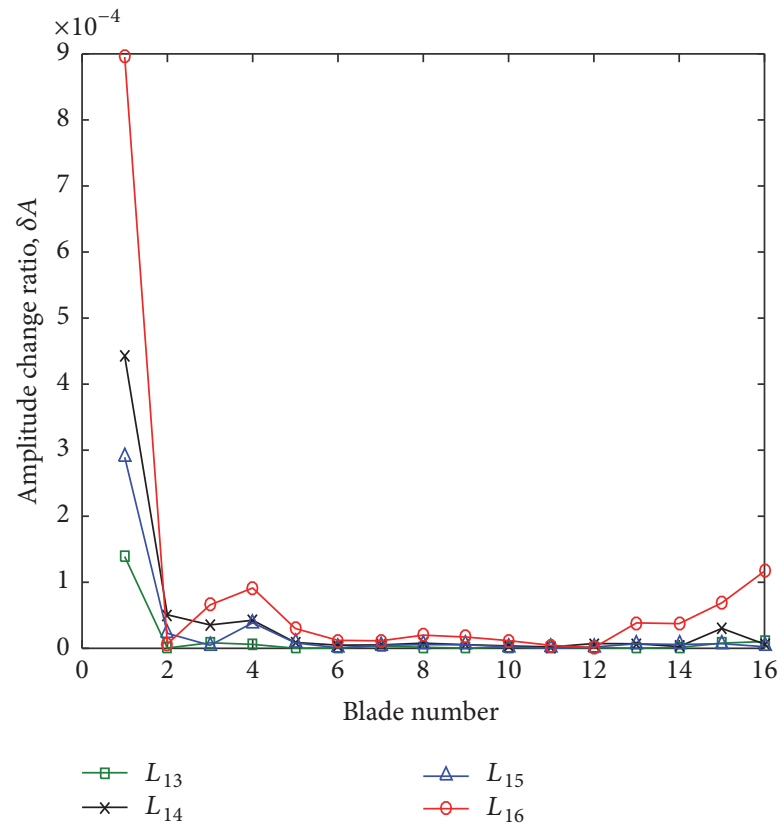

FIGURE 17: Vibration amplitude changes of MRBs under different $l c$.

changing. Then, we further explore and validate nonlinear dynamic characteristics of the cracked blade under different sizes of a breathing crack.

According to Figure 18, set $l c=85 \mathrm{~mm}$ and other parameters are fixed as in the above. Bifurcation diagram is calculated by resampling vibration displacement of the cracked blade and it is shown in Figure 19. We can see that the depth $a$ has obvious effects on nonlinear dynamic responses of the cracked blade. With increase of $a$, the following bifurcation phenomenon can be observed in Figure 19: (i) when $a$ changes from $0 \mathrm{~mm}$ to $1.43 \mathrm{~mm}$, the response of vibration displacement is a single-period motion. (ii) When $a$ changes from $1.43 \mathrm{~mm}$ to $1.65 \mathrm{~mm}$, the response of vibration displacement is a triple-period motion. (iii) When $a$ changes 


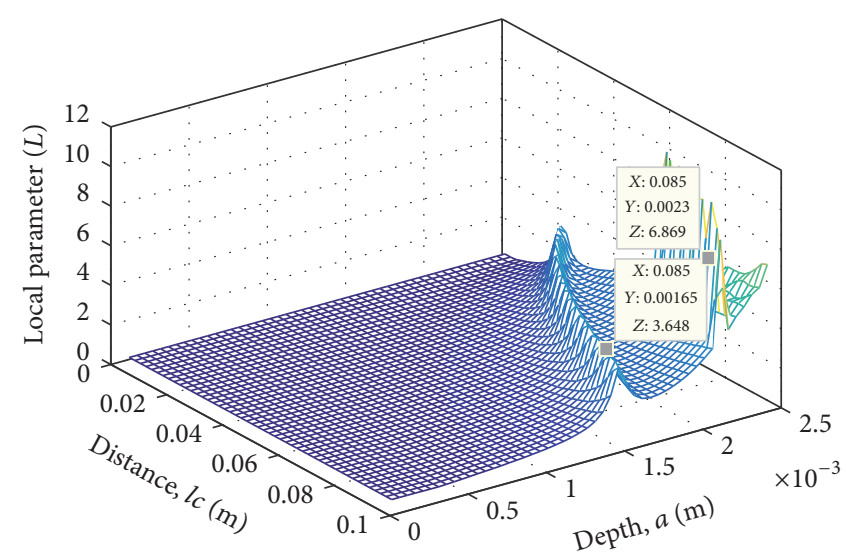

FIGURE 18: Relations between LPVRs and parameter changes of a crack.

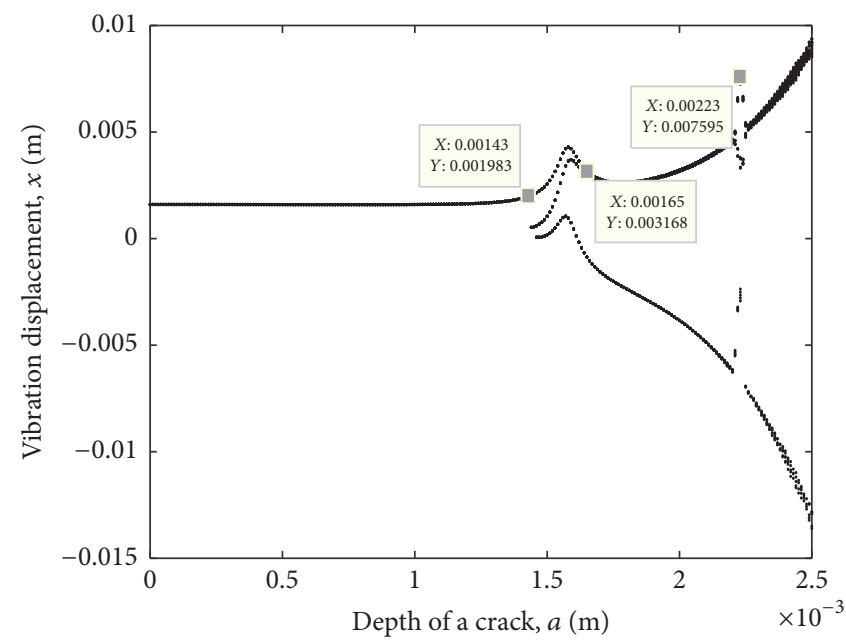

FIGURE 19: Bifurcation diagram with depth of a breathing crack.

from $1.65 \mathrm{~mm}$ to $2.23 \mathrm{~mm}$, the response of vibration displacement is a double-period motion. (iv) When $a$ is $2.23 \mathrm{~mm}$, the response of vibration displacement changes to multiperiod motion suddenly. (v) When a changes from $2.23 \mathrm{~mm}$ to $2.5 \mathrm{~mm}$, the response of vibration displacement is a doubleperiod motion again. So when $a$ is $1.65 \mathrm{~mm}$ or $2.23 \mathrm{~mm}$, motion changes suddenly, which makes peak value appear in increased process of LPVRs.

\section{Conclusions}

Diagnosis of crack faults in MRBs is very significant for safety, reliability, and availability. In practice, mistuning is inherent feature in rotated blades, which makes cracks be detected difficultly. Thus, studying the effects of crack on vibration characteristics of MRBs can provide a promising way for crack detection in MRBs. In this paper, MRBs are simplified to a coupled lumped-parameter model. Then, mistuned stiffness is introduced to represent mistuning of rotated blades, which complies with normal distribution. In addition, a breathing crack model is adopted to study effects of a crack on vibration characteristics of MRBs. Finally, eigenvalue analysis and numerical analysis are done, and the following conclusions can be drawn: (i) Mistuning causes vibration localization in MRBs. Mode localization of MRBs increases along with mistuning strengthening. In addition, intensive coupling can reduce mode localization of MRBs. (ii) Effects of depths and positions of a crack on natural frequencies and vibration amplitudes are studied. The results show that a crack causes natural frequency decease and vibration amplitude increase of cracked blade. We can use variational factors of natural frequencies and vibration amplitudes under different status of a crack to detect a crack in MRBs. (iii) Bifurcations of vibration displacement will occur due to a breathing crack, which causes nonlinear behaviors of LPVRs.

Finally, it must be noted that online crack detection in MRBs is an extreme complex problem, which is realized difficultly. In this paper, lumped-parameter model of MRBs with a breathing crack is built and effects are revealed by theoretical and numerical analyses, which provide theoretical 
guidance for crack detection in MRBs. Hence, in future study, the proposed method is expected to be used in practical engineering, which achieves online crack detection in MRBs.

\section{Competing Interests}

The authors declare that there are no competing interests regarding the publication of this paper.

\section{Acknowledgments}

This work was supported by the National Basic Research Program of China (Grant no. 2015 CB057400).

\section{References}

[1] M. Witoś, "High sensitive methods for health monitoring of compressor blades and fatigue detection," The Scientific World Journal, vol. 2013, Article ID 218460, 31 pages, 2013.

[2] C. Zhongsheng, Y. Yongmin, G. Bin, and H. Zheng, "Blade damage prognosis based on kernel principal component analysis and grey model using subsampled tip-timing signals," Proceedings of the Institution of Mechanical Engineers, Part C: Journal of Mechanical Engineering Science, vol. 228, no. 17, pp. 3178-3185, 2014.

[3] J. Lin, Z. Hu, Z.-S. Chen, Y.-M. Yang, and H.-L. Xu, "Sparse reconstruction of blade tip-timing signals for multi-mode blade vibration monitoring," Mechanical Systems and Signal Processing, vol. 81, pp. 250-258, 2016.

[4] J. H. Kuang and B. W. Huang, "Mode localization of a cracked blade disk," Journal of Engineering for Gas Turbines and Power, vol. 121, no. 2, pp. 335-341, 1999.

[5] B.-W. Huang, "Effect of number of blades and distribution of cracks on vibration localization in a cracked pre-twisted blade system," International Journal of Mechanical Sciences, vol. 48, no. 1, pp. 1-10, 2006.

[6] S.-T. Wei and C. Pierre, "Localization phenomena in mistuned assemblies with cyclic symmetry. Part I: free vibrations," Journal of Vibration, Acoustics, Stress, and Reliability in Design, vol. 110, no. 4, pp. 429-438, 1988.

[7] M. P. Castanier and C. Pierre, "Modeling and analysis of mistuned bladed disk vibration: current status and emerging directions," Journal of Propulsion and Power, vol. 22, no. 2, pp. 384-396, 2006.

[8] J.-T. Kim and N. Stubbs, "Crack detection in beam-type structures using frequency data," Journal of Sound and Vibration, vol. 259, no. 1, pp. 145-160, 2003.

[9] M. T. Piovan and R. Sampaio, "A study on the dynamics of rotating beams with functionally graded properties," Journal of Sound and Vibration, vol. 327, no. 1-2, pp. 134-143, 2009.

[10] M. A. Prohl, "A method for calculating vibration frequency and stress of a banded group of turbine buckets," Transactions of the ASME, vol. 80, no. 1, pp. 169-180, 1958.

[11] G. R. Bajaj, Free vibration of packeted turbine blades-coupled bending-bending-torsion modes [M.S. thesis], Indian Institute of Technology, Kanpur, India, 1974.

[12] H. S. Lim, J. T. Chung, and H. H. Yoo, "Modal analysis of a rotating multi-packet blade system," Journal of Sound and Vibration, vol. 325, no. 3, pp. 513-531, 2009.
[13] A. Chandrashaker and S. Adhikari, "Vibration localization of rotationally periodic structures," in Proceedings of the 10th International Conference on Vibration Engineering and Technology of Machinery (VETOMAC '14), vol. 23, pp. 865-878, September 2014.

[14] H. Wang, E. He, and Z. Zhao, "Investigating further resonant response characteristics of disk with randomly mistuned blades," Journal of Northwestern Polytechnical University, vol. 29, no. 2, pp. 189-193, 2011.

[15] T. Hai, J.-J. Wang, and Q.-H. Li, "Mistuning characteristics analysis of mistuned bladed disk assemblies," Journal of Aerospace Power, vol. 25, no. 1, pp. 160-168, 2010.

[16] J. F. Hou, "Cracking-induced mistuning in bladed disks," AIAA Journal, vol. 44, no. 11, pp. 2542-2546, 2006.

[17] J. S. Rao, Turbomachine Blade Vibration, New Age International, Delhi, India, 2010.

[18] S. S. Rao, Mechanical Vibration, Addison-Wesley, New York, NY, USA, 4th edition, 1995.

[19] S. M. Cheng, A. S. J. Swamidas, X. J. Wu, and W. Wallace, "Vibrational response of a beam with a breathing crack," Journal of Sound and Vibration, vol. 225, no. 1, pp. 201-208, 1999.

[20] H. Xu, Z. Chen, Y. Xiong, Y. Yang, and L. Tao, "Nonlinear dynamic behaviors of rotated blades with small breathing cracks based on vibration power flow analysis," Shock and Vibration, vol. 2016, Article ID 4197203, 11 pages, 2016.

[21] H. T. Liao, J. J. Wang, J. Y. Yao, and Q. H. Li, "Mistuning forced response characteristics analysis of mistuned bladed disks," Journal of Engineering for Gas Turbines and Power, vol. 132, no. 12, Article ID 122501, 11 pages, 2010.

[22] T. G. Chondros, A. D. Dimarogonas, and J. Yao, "Vibration of a beam with a breathing crack," Journal of Sound and Vibration, vol. 239, no. 1, pp. 57-67, 2001.

[23] T. Miyakozawa, R. E. Kielb, and K. C. Hall, "The effects of aerodynamic asymmetric perturbations on forced response of bladed disks," Journal of Turbomachinery, vol. 131, no. 4, 8 pages, 2009.

[24] J. A. Beck, J. A. Justice, O. E. Scott-Emuakpor, T. J. George, and J. M. Brown, "Next generation traveling-wave excitation system for integrally bladed rotors," Journal of Aerospace Engineering, vol. 28, no. 6, Article ID 04015005, 2015.

[25] H. J. Wang, Investigation of the vibration localization problem of the mistuned bladed disk with complicated coupling [Ph.D. thesis], Northwestern Potyteehnieal University, Xi'an, China, 2006.

[26] M. Huang, J. K. Liu, and Z. R. Lu, "On mode localization of a weakly coupled beam system with spring-mass attachments," Structural Engineering and Mechanics, vol. 42, no. 1, pp. 13-24, 2012.

[27] S. Kwon and H. H. Yoo, "Mode and transient response localization occurred in rotating multi-packet blade systems due to random mistuning," International Journal of Precision Engineering and Manufacturing, vol. 16, no. 10, pp. 2063-2071, 2015.

[28] Y. J. Chan, Variability of blade vibration in mistuning bladed discs [Ph.D. thesis], University of London, London, UK, 2009. 


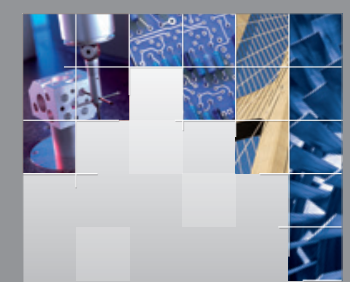

\section{Enfincering}
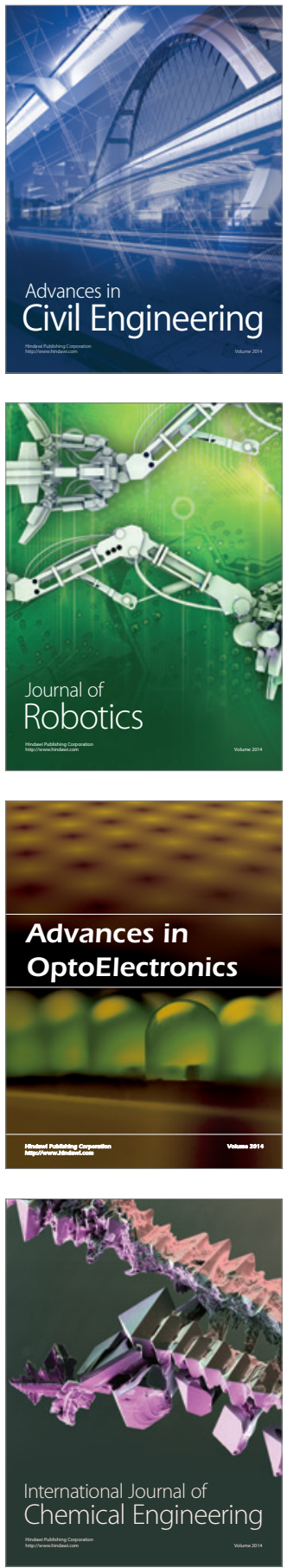

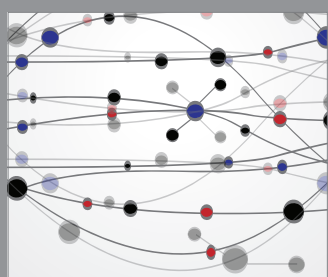

The Scientific World Journal

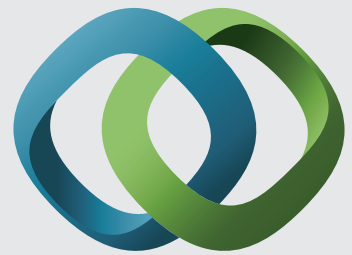

\section{Hindawi}

Submit your manuscripts at

https://www.hindawi.com
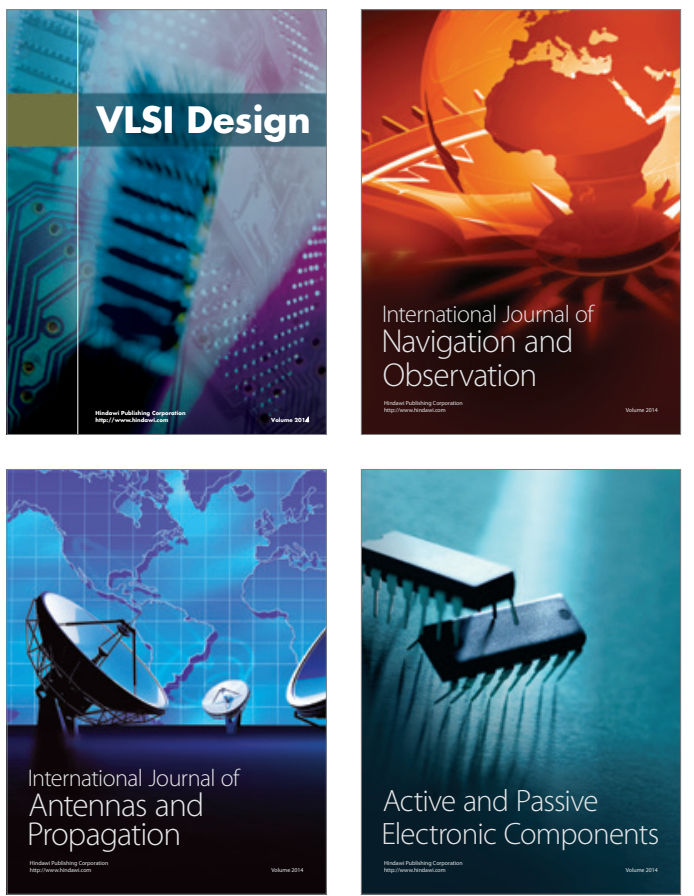
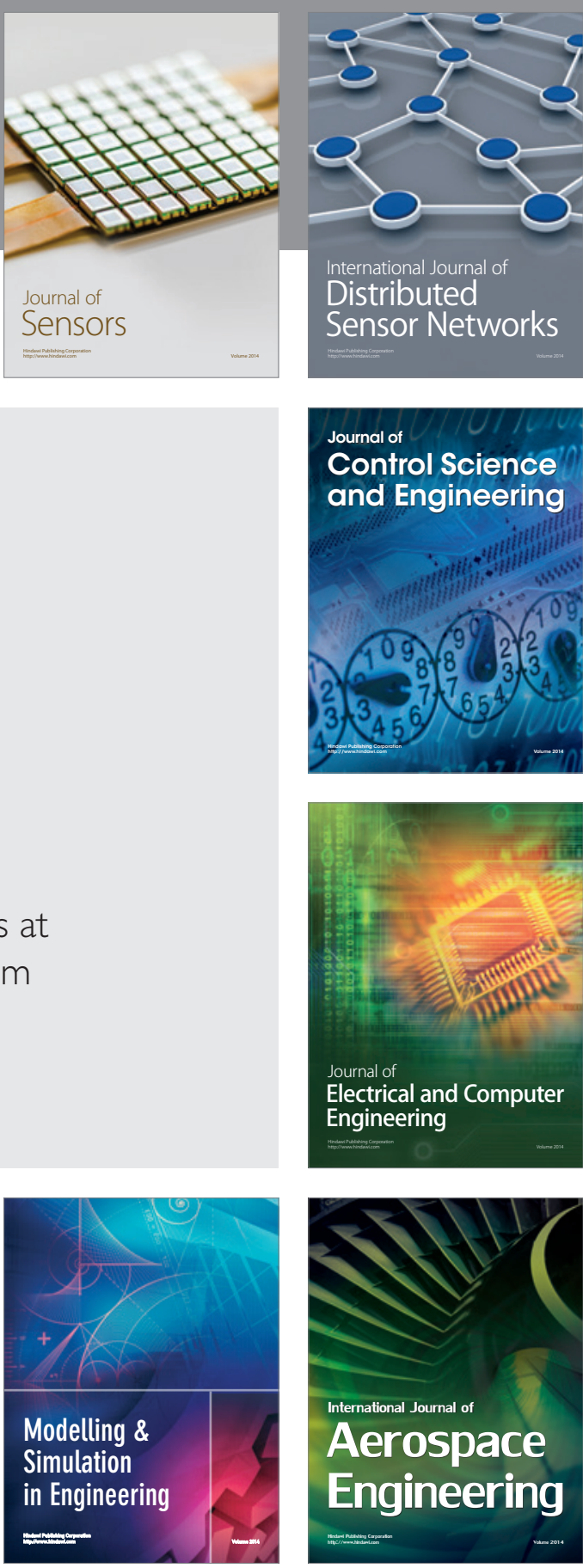

International Journal of

Distributed

Sensor Networks

$-$

Joumal of

Control Science

and Engineering
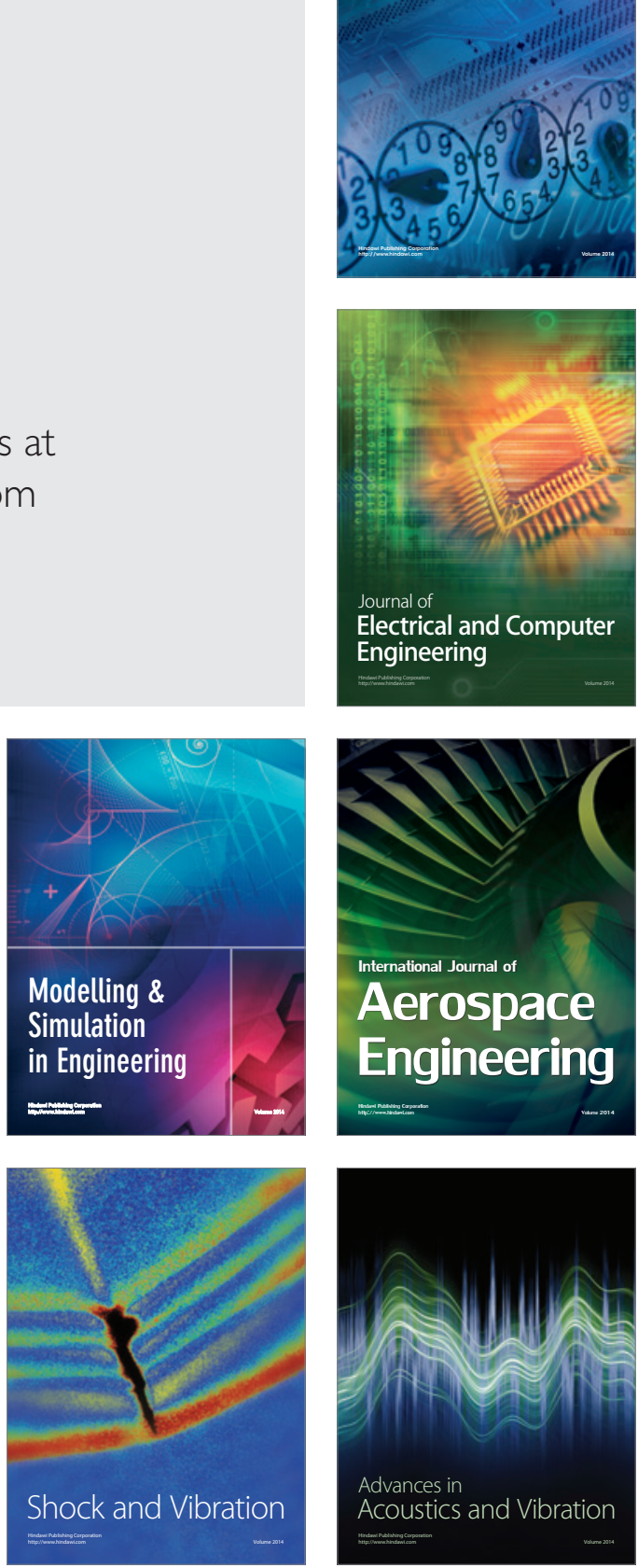